\title{
Approximating the coefficients in semilinear stochastic partial differential equations
}

\author{
MARKUS KUNZE AND JAN VAN NEERVEN
}

Abstract. We investigate, in the setting of UMD Banach spaces $E$, the continuous dependence on the data $A, F, G$ and $\xi$ of mild solutions of semilinear stochastic evolution equations with multiplicative noise of the form

$$
\left\{\begin{array}{l}
\mathrm{d} X(t)=[A X(t)+F(t, X(t))] \mathrm{d} t+G(t, X(t)) \mathrm{d} W_{H}(t), \quad t \in[0, T], \\
X(0)=\xi
\end{array}\right.
$$

where $W_{H}$ is a cylindrical Brownian motion in a Hilbert space $H$. We prove continuous dependence of the compensated solutions $X(t)-e^{t A} \xi$ in the norms $L^{p}\left(\Omega ; C^{\lambda}([0, T] ; E)\right)$ assuming that the approximating operators $A_{n}$ are uniformly sectorial and converge to $A$ in the strong resolvent sense and that the approximating nonlinearities $F_{n}$ and $G_{n}$ are uniformly Lipschitz continuous in suitable norms and converge to $F$ and $G$ pointwise. Our results are applied to a class of semilinear parabolic SPDEs with finite dimensional multiplicative noise.

\section{Introduction}

We consider semilinear stochastic evolution equations with multiplicative noise of the form

$$
\left\{\begin{array}{l}
d X(t)=[A X(t)+F(t, X(t))] \mathrm{d} t+G(t, X(t)) \mathrm{d} W_{H}(t), \quad t \in[0, T], \\
X(0)=\xi
\end{array}\right.
$$

where $A$ is the generator of a strongly continuous analytic semigroup $\mathbf{S}=(S(t))_{t \geq 0}$ on a UMD Banach space $E$, the driving process $W_{H}$ is a cylindrical Brownian motion in a Hilbert space $H$ defined on some probability space $\Omega$ (see Sect. 3 for the definition), the functions $F:[0, T] \times \Omega \times E \rightarrow E$ and $G:[0, T] \times \Omega \times E \rightarrow \mathscr{L}(H, E)$ satisfy suitable measurability and Lipschitz continuity conditions, and the initial value $\xi$ is an $E$-valued random variable on $\Omega$.

The theory of stochastic integration in UMD Banach spaces yields existence, uniqueness, and regularity of mild solutions [26,27]. It is a natural question how this solution depends on the 'coefficients' $A, F, G$ and the initial datum $\xi$. Our main abstract results are Theorems 4.3 and 4.7 which assert, roughly speaking, that the

The authors are supported by VICI subsidy 639.033.604 of the Netherlands Organisation for Scientific Research (NWO). 
solution $X(\cdot)$ and the compensated solution $X(\cdot)-S(\cdot) \xi$ depends continuously on $A, F, G$ and $\xi$ simultaneously with respect to the norms of $L^{p}(\Omega ; C([0, T] ; E))$ and $L^{p}\left(\Omega ; C^{\lambda}([0, T] ; E)\right)$, respectively.

In the case when $E$ is a Hilbert space, concerning dependence of the solution on the initial datum $\xi$, we refer to Da Prato and Zabczyk [9]; see also the recent work by Marinelli, Prévôt, and Röckner [23] for the case of Poisson noise. Approximations of the functions $F$ and $G$ are considered in Peszat and Zabczyk [29] and Seidler [30]. Under more restrictive assumptions than ours, simultaneous approximations of $A, F, G$, and $\xi$ were considered by Brzeźniak [5] in the setting of UMD Banach spaces with type 2 .

These approximation results provide a justification for the use of numerical schemes, where necessarily one replaces continuous objects by discretized approximations. Furthermore, approximating $A$ by bounded operators $A_{n}$ (such as their Yosida approximations, see Sect. 5.2) is often helpful on a technical level, for instance in the standard proofs of the Itô lemma in infinite dimensions [6,9].

We apply our abstract results to the stochastic partial differential equation (SPDE)

$$
\left\{\begin{array}{lll}
\frac{\partial u}{\partial t}(t, x)=\mathcal{A} u(t, x)+f(u(t, x))+\sum_{k=1}^{K} g_{k}(u(t, x)) \frac{\partial W_{k}}{\partial t}(t), & x \in \mathcal{O}, & t \geq 0, \\
u(t, x)=0, & x \in \partial \mathcal{O}, t \geq 0, \\
u(0, x)=\xi(x), & x \in \mathcal{O} .
\end{array}\right.
$$

Here, $\mathcal{O}$ is a bounded open domain in $\mathbb{R}^{d}$ and

$$
\mathcal{A} u(x)=\sum_{i=1}^{d} \frac{\partial}{\partial x_{i}}\left(a_{i j}(x) \sum_{j=1}^{d} \frac{\partial u}{\partial x_{j}}(x)\right)+\sum_{j=1}^{d} b_{j}(x) \frac{\partial u}{\partial x_{j}}(x)
$$

is a second-order differential operator in divergence form whose coefficients $\mathbf{a}=\left(a_{i j}\right)$ and $\mathbf{b}=\left(b_{j}\right)$ satisfy suitable boundedness and uniform ellipticity conditions. The functions $f$ and $g_{k}$ are Lipschitz continuous, and the driving processes $W_{k}$ are independent real-valued standard Brownian motions. For this problem, our abstract results imply the following approximation result. We let $\|f\|_{\text {Lip }}=\sup _{t \neq s} \frac{|f(t)-f(s)|}{|t-s|}$ denote the Lipschitz seminorm of a function $f$.

THEOREM 1.1. Let $\mathbf{a}, \mathbf{a}_{n} \in L^{\infty}\left(\mathcal{O} ; \mathbb{R}^{d \times d}\right)$, let $\mathbf{b}, \mathbf{b}_{n} \in L^{\infty}\left(\mathcal{O} ; \mathbb{R}^{d}\right)$, and let $f, f_{n}, g_{k}, g_{k, n}: \mathbb{R} \rightarrow \mathbb{R}$ be Lipschitz continuous. Assume that there exist finite constants $\kappa, C>0$ such that:

(i) $\mathbf{a}, \mathbf{a}_{n}$ are symmetric and $\mathbf{a x} \cdot \mathbf{x}, \mathbf{a}_{n} \mathbf{x} \cdot \mathbf{x} \geq \kappa|\mathbf{x}|^{2}$ for all $\mathbf{x} \in \mathbb{R}^{d}$;

(ii) $\|\mathbf{a}\|_{\infty},\left\|\mathbf{a}_{n}\right\|_{\infty},\|\mathbf{b}\|_{\infty},\left\|\mathbf{b}_{n}\right\|_{\infty} \leq C$;

(iii) $\|f\|_{\text {Lip }},\left\|f_{n}\right\|_{\text {Lip }},\left\|g_{k}\right\|_{\text {Lip }},\left\|g_{k, n}\right\|_{\text {Lip }} \leq C$.

Assume further that

(iv) $\lim _{n \rightarrow \infty} \mathbf{a}_{n}=\mathbf{a}, \lim _{n \rightarrow \infty} \mathbf{b}_{n}=\mathbf{b}$ almost everywhere on $\mathcal{O}$;

(v) $\lim _{n \rightarrow \infty} f_{n}=f, \lim _{n \rightarrow \infty} g_{k, n}=g_{k}$ pointwise on $\mathcal{O}$. 
Let $1<p<\infty$. If $\xi_{n} \rightarrow \xi$ in $L^{p}(\mathcal{O})$, the approximate mild solutions $u_{n}$ converge to the mild solution $u$ in the following compensated sense: for all $1 \leq q<\infty$ and $0 \leq \lambda<\frac{1}{2}$ we have

$$
u_{n}-S_{n}(\cdot) \xi_{n} \rightarrow u-S(\cdot) \xi \text { in } L^{q}\left(\Omega ; C^{\lambda}\left([0, T] ; L^{p}(\mathcal{O})\right)\right) .
$$

Here, $S_{n}(\cdot)$ and $S(\cdot)$ denote the strongly continuous analytic semigroups generated by the $L^{p}(\mathcal{O})$-realizations of $\mathcal{A}_{n}$ and $\mathcal{A}$. Furthermore, for all $1 \leq q<\infty$, we have $u_{n} \rightarrow u$ in $L^{q}\left(\Omega ; C\left([0, T] ; L^{p}(\mathcal{O})\right)\right)$.

A slightly more general version of this theorem allowing for random initial conditions is presented below (Theorem 5.3). It is interesting to compare this result with Krylov's [19, Theorem 5.7] where, for elliptic operators in nondivergence form, convergence in a stronger norm is obtained under a stronger convergence assumption on the functions $g_{k}$.

It is possible to extend our results to SPDEs with locally Lipschitz continuous nonlinearities, measurable initial values, and infinite dimensional noise; also, regularity in both space and time can be accounted for. These extensions involve the use of interpolation techniques and require additional assumptions on the domains $\mathrm{D}(A)$ and $\mathrm{D}\left(A_{n}\right)$. In order to keep this article at a reasonable length, we have chosen to postpone these extensions to a forthcoming publication [21].

The organization of the paper is as follows. In Sect. 2, we prove an abstract approximation result for certain spaces of $\gamma$-radonifying operators. After recalling some results about solving the abstract problem (SCP) in Sect. 3, we prove our main abstract approximation results in Sect. 4. Theorem 1.1 is proved in Sect. 5, where some further applications are presented as well.

Throughout this article, all vector spaces are real. Whenever this is needed, e.g., when using spectral theory, we shall pass to their complexifications. We assume the reader to be familiar with standard Banach space concepts such as the UMD property and the notions of type and cotype. For more information, we recommend the survey articles by Burkholder and Maurey in the Handbook of Geometry of Banach Spaces $[15,16]$.

When $P_{n}(\phi)$ and $Q_{n}(\phi)$ are certain quantities depending on an index $n$ and a function $\phi$, we use the notation $P_{n}(\phi) \lesssim Q_{n}(\phi)$ to indicate that there is a constant $C$, independent of $\phi$, such that $P_{n}(\phi) \leq C Q_{n}(\phi)$ holds for all indices $n$. Unless otherwise stated, this constant is allowed to depend on all other relevant data. We write $P_{n}(\phi) \approx Q_{n}(\phi)$ if $P_{n}(\phi) \lesssim Q_{n}(\phi)$ and $Q_{n}(\phi) \lesssim P_{n}(\phi)$.

\section{Approximation of $\gamma$-radonifying operators}

We begin with a brief discussion of spaces of $\gamma$-radonifying operators, which play an important role in the theory of stochastic integration in UMD Banach spaces.

Let $\mathscr{H}$ be a Hilbert space (below we shall take $\mathscr{H}=L^{2}(0, T ; H)$, where $H$ is another Hilbert space) and $E$ be a Banach space. Any finite rank operator $R: \mathscr{H} \rightarrow E$ 
can be represented in the form $\sum_{n=1}^{N} h_{n} \otimes x_{n}$, where the vectors $h_{n}$ are orthonormal in $\mathscr{H}$ and the vectors $x_{n}$ belong to $E$. For such an operator, we define

$$
\|R\|_{\gamma(\mathscr{H}, E)}^{2}:=\mathbb{E}\left\|\sum_{n=1}^{N} \gamma_{n} R h_{n}\right\|^{2} .
$$

Here, and in what follows, $\left(\gamma_{n}\right)_{n=1}^{N}$ is a sequence of independent real-valued standard Gaussian random variables.

It is easy to check that the above identity defines a norm on the space $\mathscr{H} \otimes E$ of all finite rank operators from $\mathscr{H}$ to $E$. The completion of $\mathscr{H} \otimes E$ with respect to this norm is denoted by $\gamma(\mathscr{H}, E)$. This space is contractively embedded into $\mathscr{L}(\mathscr{H}, E)$. A bounded operator in $\mathscr{L}(\mathscr{H}, E)$ is called $\gamma$-radonifying if it belongs to $\gamma(\mathscr{H}, E)$.

For all $R \in \gamma(\mathscr{H}, E)$, we have the identity

$$
\|R\|_{\gamma(\mathscr{H}, E)}^{2}=\sup _{h} \mathbb{E}\left\|\sum_{j=1}^{k} \gamma_{j} R h_{j}\right\|^{2},
$$

where the supremum is taken over all finite orthonormal systems $h=\left\{h_{j}\right\}_{j=1}^{k}$ in $\mathscr{H}$. A bounded operator $R$ from $\mathscr{H}$ to $E$ is called $\gamma$-summing if the above supremum is finite. This supremum, denoted by $\|R\|_{\gamma_{\infty}(\mathscr{H}, E)}$, turns the space of all $\gamma$-summing operators from $\mathscr{H}$ to $E$ into a Banach space. By definition, we have an isometric inclusion $\gamma(\mathscr{H}, E) \subseteq \gamma_{\infty}(\mathscr{H}, E)$. It follows from a result of Hoffmann-Jørgensen and Kwapień $[14,22]$ that $\gamma(\mathscr{H}, E)=\gamma_{\infty}(\mathscr{H}, E)$ if (and only if) $E$ does not contain a closed subspace isomorphic to $c_{0}$.

The space $\gamma(\mathscr{H}, E)$ enjoys the following ideal property: if $T \in \mathscr{L}\left(\mathscr{H}_{2}, \mathscr{H}_{1}\right)$, $S \in \mathscr{L}\left(E_{1}, E_{2}\right)$ and $R \in \gamma\left(\mathscr{H}_{1}, E_{1}\right)$, where $\mathscr{H}_{1}, \mathscr{H}_{2}$ are Hilbert spaces and $E_{1}, E_{2}$ are Banach spaces, then $S R T \in \gamma\left(\mathscr{H}_{2}, E_{2}\right)$ and

$$
\|S R T\|_{\gamma\left(\mathscr{H}_{2}, E_{2}\right)} \leq\|S\|_{\mathscr{L}\left(E_{1}, E_{2}\right)}\|R\|_{\gamma\left(\mathscr{H}_{1}, E_{1}\right)}\|T\|_{\mathscr{L}\left(\mathscr{H}_{2}, \mathscr{H}_{1}\right)}
$$

The analogous result holds for the space $\gamma_{\infty}(\mathscr{H}, E)$.

For more information and proofs, we refer to the review article [25] and the references given therein.

We are mainly interested in the case $\mathscr{H}=L^{2}(X, \mu ; H)$, where $\mu$ is a $\sigma$-finite measure on some measurable space $X$ and $H$ is another Hilbert space. In this situation, we say that a function $\Phi: X \mapsto \mathscr{L}(H, E)$ represents a bounded operator $R: L^{2}(X, \mu ; H) \rightarrow E$ if the following two conditions are satisfied:

(i) For all $x^{*} \in E^{*}$, the function $t \mapsto \Phi^{*}(t) x^{*}$ belongs to $L^{2}(X, \mu ; H)$;

(ii) For all $f \in L^{2}(X, \mu ; H)$ and $x^{*} \in E^{*}$, we have

$$
\left\langle R f, x^{*}\right\rangle=\int_{X}\left[f(t), \Phi^{*}(t) x^{*}\right] \mathrm{d} \mu(t) .
$$


If $t \mapsto \Phi(t) h$ is strongly measurable for all $h \in H$, then the operator $R$ is uniquely determined by $\Phi$; see [27].

It will be important to have criteria for checking whether a given function $\Phi: X \rightarrow$ $\mathscr{L}(H, E)$ represents an operator in the space $\gamma\left(L^{2}(X, \mu ; H), E\right)$. We begin with the following simple result; see [12].

PROPOSITION 2.1. For all $f \in L^{2}(X ; \mu)$ and $S \in \gamma(H, E)$, the function $f \otimes S$ : $t \mapsto f(t) S$ represents a unique operator $R_{f \otimes S} \in \gamma\left(L^{2}(X, \mu ; H), E\right)$, which is given by

$$
R_{f \otimes S} g=\int_{X} f(t) S g(t) \mathrm{d} \mu(t)
$$

and we have

$$
\left\|R_{f \otimes S}\right\|_{\gamma\left(L^{2}(X, \mu ; H), E\right)}=\|f\|_{L^{2}(X, \mu)}\|S\|_{\gamma(H, E)} .
$$

The following sufficient condition for a function $\Phi:(a, b) \rightarrow \mathscr{L}(H, E)$ to represent an element of $\gamma\left(L^{2}(a, b ; H), E\right)$ is a simple extension of a result due to Kalton and Weis [18]. For the proof, we refer to [25, Proposition 13.9].

PROPOSITION 2.2. Let $\Phi:(a, b) \rightarrow \gamma(H, E)$ be continuously differentiable with

$$
\int_{a}^{b}(t-a)^{\frac{1}{2}}\left\|\Phi^{\prime}(t)\right\|_{\gamma(H, E)} \mathrm{d} t<\infty
$$

Then $\Phi$ represents a unique operator $R_{\Phi} \in \gamma\left(L^{2}(a, b ; H), E\right)$ and

$$
\left\|R_{\Phi}\right\|_{\gamma\left(L^{2}(a, b ; H), E\right)} \leq(b-a)^{\frac{1}{2}}\|\Phi(b-)\|_{\gamma(H, E)}+\int_{a}^{b}(t-a)^{\frac{1}{2}}\left\|\Phi^{\prime}(t)\right\|_{\gamma(H, E)} \mathrm{d} t .
$$

A subset $\mathscr{T} \subseteq \mathscr{L}(E, F)$, where $E, F$ are Banach spaces, is called $\gamma$-bounded, if there exists $C \geq 0$ such that for all finite sequences $x_{1}, \ldots, x_{N} \in E$ and $T_{1}, \ldots, T_{N} \in$ $\mathscr{T}$, we have

$$
\mathbb{E}\left\|\sum_{n=1}^{N} \gamma_{n} T_{n} x_{n}\right\|^{2} \leq C^{2} \mathbb{E}\left\|\sum_{n=1}^{N} \gamma_{n} x_{n}\right\|^{2}
$$

The infimum over all admissible constants $C$ is called the $\gamma$-bound of $\mathscr{T}$ and is denoted by $\gamma(\mathscr{T})$. For more information on $\gamma$-boundedness and the related notion of $R$-boundedness, we refer to [7] and the lecture notes [20]. We will need the following elementary fact.

PROPOSITION 2.3. If $\mathscr{T}$ is $\gamma$-bounded, then the closure in the strong operator topology of its absolute convex hull is $\gamma$-bounded as well, and $\gamma(\overline{\mathrm{co}}(\mathscr{T}))=\gamma(\mathscr{T})$.

We will also need the following sufficient condition for $\gamma$-boundedness due to Weis [31]. 
PROPOSITION 2.4. Suppose $\Phi:(a, b) \rightarrow \mathscr{L}(H, E)$ is continuously differentiable. If $\Phi^{\prime}$ is integrable, then the family $\mathscr{T}_{\Phi}:=\{\Phi(t): t \in(a, b)\}$ is $\gamma$-bounded and

$$
\gamma\left(\mathscr{T}_{\Phi}\right) \leq\|\Phi(a+)\|+\int_{a}^{b}\left\|\Phi^{\prime}(t)\right\| \mathrm{d} t .
$$

In order to be able to state a second sufficient condition for being a member of $\gamma\left(L^{2}(a, b ; H), E\right)$, we need to make a simple preliminary observation. Let $\Phi:(a, b) \rightarrow \mathscr{L}(H, E)$ be a function of the form

$$
\Phi=\sum_{m=1}^{M} f_{m} \otimes\left(h_{m} \otimes x_{m}\right),
$$

with $f_{m} \in L^{2}(a, b), h_{m} \in H, x_{m} \in E$. Then $\Phi$ represents the operator

$$
R_{\Phi}=\sum_{m=1}^{M}\left(f_{m} \otimes h_{m}\right) \otimes x_{m}
$$

which is of finite rank and therefore belongs to $\gamma\left(L^{2}(a, b ; H), E\right)$. It will be important later on that the linear span of all such operators is dense in $\gamma\left(L^{2}(a, b ; H), E\right)$; see [28].

Now, let $F$ be a second Banach space and suppose that $M:(a, b) \rightarrow \mathscr{L}(E, F)$ is a function with the property that $t \mapsto M(t) x$ is strongly measurable and bounded for all $x \in E$. If $\Phi$ is as above, then the function $M \Phi: t \mapsto M(t) \Phi(t)$ is strongly measurable and represents a unique bounded operator $R_{M \Phi}$ from $L^{2}(a, b ; H)$ to $F$.

Under these assumptions, one has the following result, also due to Kalton and Weis [18], a proof can be found in [25].

PROPOSITION 2.5. Let $E$ and $F$ be Banach spaces and suppose $\Phi$ represents an operator $R_{\Phi} \in \gamma\left(L^{2}(a, b ; H), E\right)$. If $M$ has $\gamma$-bounded range $\mathscr{M}=\{M(t): t \in$ $(a, b)\}$, then $R_{M \Phi} \in \gamma_{\infty}\left(L^{2}(a, b ; H), F\right)$ and

$$
\left\|R_{M \Phi}\right\|_{\gamma_{\infty}\left(L^{2}(a, b ; H), F\right)} \leq \gamma(\mathscr{M})\left\|R_{\Phi}\right\|_{\gamma\left(L^{2}(a, b ; H), E\right)} .
$$

Consequently, the mapping $R_{\Phi} \mapsto R_{M \Phi}$ has a unique extension to a bounded linear operator (also denoted by $M)$ from $\gamma\left(L^{2}(a, b ; H), E\right)$ to $\gamma_{\infty}\left(L^{2}(a, b ; H), F\right)$ of norm at most $\gamma(\mathscr{M})$.

In our main results, we shall always assume that $E=F$ is a UMD space. Such spaces, being reflexive, cannot contain isomorphic copies of $c_{0}$, and therefore, $\gamma_{\infty}\left(L^{2}(a, b ; H), F\right)=\gamma\left(L^{2}(a, b ; H), F\right)$ in this situation.

After these preparations, we are in a position to state the main approximation lemma of this section.

LEMMA 2.6. Let $E$ and $F$ be Banach spaces. Let the functions $M_{n}, M:(a, b) \rightarrow$ $\mathscr{L}(E, F)$ satisfy the following conditions: 
(1) For all $x \in E$, the functions $M_{n}(\cdot) x$ and $M(\cdot) x$ are continuously differentiable on $(a, b)$;

(2) For all $x \in E$, we have $\lim _{n \rightarrow \infty} M_{n}(t) x=M(t) x$ and $\lim _{n \rightarrow \infty} M_{n}^{\prime}(t) x=$ $M^{\prime}(t) x$ uniformly on compact subsets of $(a, b)$;

(3) The sets $\mathscr{M}_{n}=\left\{M_{n}(t): t \in(a, b)\right\}$ are $\gamma$-bounded and $\sup _{n} \gamma\left(\mathscr{M}_{n}\right)<\infty$.

Then for all $R \in \gamma\left(L^{2}(a, b ; H), E\right)$, we have $M_{n} R, M R \in \gamma\left(L^{2}(a, b ; H), F\right)$ and

$$
\lim _{n \rightarrow \infty} M_{n} R=M R \text { in } \gamma\left(L^{2}(a, b ; H), F\right) .
$$

Here, the operators $M_{n}, M: \gamma\left(L^{2}(a, b ; H), E\right) \rightarrow \gamma_{\infty}\left(L^{2}(a, b ; H), F\right)$ are as in Proposition 2.5.

Proof. First, we consider the case where $R$ is represented by the function $\mathbb{1}_{\left(a^{\prime}, b^{\prime}\right)} \otimes S$, where $a<a^{\prime}<b^{\prime}<b$ and $S \in \gamma(H, E)$ is a fixed finite rank operator, say $S=\sum_{j=1}^{k} h_{j} \otimes x_{j}$ with the vectors $h_{j} \in H$ orthonormal.

Proposition 2.2 (with $a, b$ replaced with $a^{\prime}, b^{\prime}$ ) implies that $M_{n}(\cdot) R$ and $M(\cdot) R$ belong to $\gamma\left(L^{2}\left(a^{\prime}, b^{\prime} ; H\right), F\right)$, and hence to $\gamma\left(L^{2}(a, b ; H), F\right)$, and by Propositions 2.5 and 2.1,

$$
\left\|M_{n}(\cdot) R\right\|_{\gamma\left(L^{2}(a, b ; H), F\right)} \leq C\left\|\mathbb{1}_{\left(a^{\prime}, b^{\prime}\right)}\right\|_{L^{2}(a, b)}\|S\|_{\gamma(H, E)} \leq C(b-a)^{\frac{1}{2}}\|S\|_{\gamma(H, E)}
$$

with $C:=\sup _{n} \gamma\left(\mathscr{M}_{n}\right)$. Taking strong limits and invoking Proposition 2.3, $\{M(t)$ : $t \in(a, b)\}$ is $\gamma$-bounded with $\gamma$-bound at most $C$, and therefore, the same estimates hold with $M_{n}$ replaced by $M$.

We claim that

$$
\lim _{n \rightarrow \infty} M_{n}(\cdot) R=M(\cdot) R \text { in } \gamma\left(L^{2}(a, b ; H), F\right) .
$$

From the representation $S=\sum_{j=1}^{k} h_{j} \otimes x_{j}$, it follows that

$$
\begin{aligned}
\left\|r^{-1}\left[M_{n}(t+r) S-M_{n}(t) S\right]-M_{n}^{\prime}(t) S\right\|_{\gamma(H, F)} \\
\quad=\left(\mathbb{E}\left\|\sum_{j=1}^{N} \gamma_{j}\left(r^{-1}\left[M_{n}(t+r)-M_{n}(t)\right]-M_{n}^{\prime}(t)\right) S h_{j}\right\|_{F}^{2}\right)^{\frac{1}{2}} \\
\quad \leq \sum_{j=1}^{N}\left\|\left(r^{-1}\left[M_{n}(t+r)-M_{n}(t)\right]-M_{n}^{\prime}(t)\right) S h_{j}\right\|_{F} \rightarrow 0 \text { as } r \rightarrow 0,
\end{aligned}
$$

for all $t \in(a, b)$ and $n \geq 1$. Hence, $M_{n}(\cdot) S$ is differentiable on $(a, b)$ as a $\gamma(H, F)$ valued function with derivative $M_{n}^{\prime}(\cdot) S$. Similarly, we see that $M(\cdot) S$ is differentiable with derivative $M^{\prime}(\cdot) S$, and arguing as above, we see that $M_{n}^{\prime}(\cdot) S$ and $M^{\prime}(\cdot) S$ are 
continuous on $(a, b)$. It now follows from Proposition 2.2 that

$$
\begin{aligned}
\left\|M_{n} R-M R\right\|_{\gamma\left(L^{2}(a, b ; H), F\right)}= & \left\|M_{n} S-M S\right\|_{\gamma\left(L^{2}\left(a^{\prime}, b^{\prime} ; H\right), F\right)} \\
\leq & \int_{a^{\prime}}^{b^{\prime}}\left(t-a^{\prime}\right)^{\frac{1}{2}}\left\|M_{n}^{\prime}(t) S-M^{\prime}(t) S\right\|_{\gamma(H, F)} \mathrm{d} t \\
& +\left(b^{\prime}-a^{\prime}\right)^{\frac{1}{2}}\left\|M_{n}\left(b^{\prime}\right) S-M\left(b^{\prime}\right) S\right\|_{\gamma(H, F)},
\end{aligned}
$$

where the integral is finite since $M_{n}^{\prime}(\cdot) S$ and $M^{\prime}(\cdot) S$ are continuous $\gamma(H, F)$-valued functions on $\left[a^{\prime}, b^{\prime}\right]$. Since $M_{n}^{\prime}(t) \rightarrow M^{\prime}(t)$ strongly for every $t \in(a, b)$ we see, using that $S$ has finite rank as before and that $M_{n}^{\prime}(t) S \rightarrow M^{\prime}(t) S$ in $\gamma(H, F)$. This convergence is uniform on $\left[a^{\prime}, b^{\prime}\right]$, and therefore, the integral in the estimate (2.1) converges to 0 . Convergence of the second term in (2.1) follows from $M_{n}\left(b^{\prime}\right) \rightarrow M\left(b^{\prime}\right)$ strongly and the fact that $S$ has finite rank. This proves the claim.

Since the multiplication operators associated with $M_{n}$ are uniformly bounded by Proposition 2.5 and assumption (3), the general case follows from a density argument. To that end, observe that the step functions with values in the finite rank operators and support in a proper two-sided subinterval $\left(a^{\prime}, b^{\prime}\right)$ of $(a, b)$ are dense in $\gamma\left(L^{2}(a, b ; H), E\right)$. This follows, e.g., from [27, Proposition 2.4].

From now on, we will no longer distinguish between a function $\Phi:(a, b) \rightarrow$ $\mathscr{L}(H, E)$ and the operator $R_{\Phi}: L^{2}(a, b ; H) \rightarrow E$ represented by it.

\section{Semilinear stochastic evolution equations}

In this section, we collect some known facts concerning the existence and uniqueness of mild solutions of the problem (SCP),

$$
\left\{\begin{array}{l}
\mathrm{d} X(t)=[A X(t)+F(t, X(t))] \mathrm{d} t+G(t, X(t)) \mathrm{d} W_{H}(t), \quad t \in[0, T], \\
X(0)=\xi
\end{array}\right.
$$

The probability space $(\Omega, \Sigma, \mathbb{P})$, endowed with a filtration $\mathbb{F}=\left(\mathscr{F}_{t}\right)_{t \geq 0}$, is fixed throughout this paper. The driving process $W_{H}: L^{2}\left(\mathbb{R}_{+} ; H\right) \rightarrow L^{2}(\Omega)$ is an $\mathbb{F}$-cylindrical Brownian motion, that is, it is a bounded linear operator from $L^{2}\left(\mathbb{R}_{+} ; H\right)$ to $L^{2}(\Omega)$ with the following properties:

(i) for all $f \in L^{2}\left(\mathbb{R}_{+} ; H\right)$, the random variable $W_{H}(f)$ is centered Gaussian

(ii) for all $t \in \mathbb{R}_{+}$and $f \in L^{2}\left(\mathbb{R}_{+} ; H\right)$ with support in $[0, t], W_{H}(f)$ is $\mathscr{F}_{t}$-measurable.

(iii) for all $t \in \mathbb{R}_{+}$and $f \in L^{2}\left(\mathbb{R}_{+} ; H\right)$ with support in $[t, \infty), W_{H}(f)$ is independent of $\mathscr{F}_{t}$.

(iv) for all $f_{1}, f_{2} \in L^{2}\left(\mathbb{R}_{+} ; H\right)$, we have $\mathbb{E}\left(W_{H}\left(f_{1}\right) \cdot W_{H}\left(f_{2}\right)\right)=\left[f_{1}, f_{2}\right]_{L^{2}\left(\mathbb{R}_{+} ; H\right)}$. It is easy to see that for all $h \in H$, the process $\left(W_{H}(t) h\right)_{t \geq 0}$ is defined by

$$
W_{H}(t) h:=W_{H}\left(\mathbb{1}_{(0, t]} \otimes h\right)
$$


is an $\mathscr{F}$-Brownian motion (which is standard if $\|h\|=1$ ). Moreover, two such Brownian motions $\left(\left(W_{H}(t) h_{1}\right)_{t \geq 0}\right.$ and $\left(\left(W_{H}(t) h_{2}\right)_{t \geq 0}\right.$ are independent if and only if $h_{1}$ and $h_{2}$ are orthogonal in $H$. We refer to [25] for a further discussion.

The linear operator $A$ is assumed to be closed and densely on $E$, and the functions $F:[0, T] \times \Omega \times E \rightarrow E$ and $G:[0, T] \times \Omega \times E \rightarrow \mathscr{L}(H, E)$ are strongly measurable and adapted and satisfy suitable Lipschitz and growth conditions specified below.

Concerning the operator $A$, we make the following assumption:

(A) The operator $A$ generates a strongly continuous analytic semigroup $\mathbf{S}=(S(t))_{t \geq 0}$ on $E$.

Recall that a closed operator $A$ generates a strongly continuous analytic semigroup on a Banach space $E$ if and only if $A$ is densely defined and sectorial, i.e., there exist $M \geq 1$ and $w \in \mathbb{R}$ such that $\{\lambda \in \mathbb{C}: \operatorname{Re} \lambda>w\}$ is contained in the resolvent set $\varrho(A)$ and

$$
\sup _{\operatorname{Re} \lambda>w}\|(\lambda-w) R(\lambda, A)\| \leq M .
$$

The constants $M$ and $w$ are called the sectoriality constants of $A$; in this context, we say that $A$ is sectorial of type $(M, w)$.

If (A) holds, then $S(t)$ maps $E$ into the domain $\mathrm{D}(A)$ and $\lim \sup _{t \downarrow 0} t\|A S(t)\|<\infty$. By Proposition 2.4, this implies the following useful fact (see, e.g., [26, Lemma 4.1]):

LEMMA 3.1. If A generates a strongly continuous analytic semigroup on a Banach space $E$, then for all $t \in[0, T]$ and $\alpha>0$ the set $\mathscr{T}_{\alpha, t}:=\left\{s^{\alpha} S(s): s \in[0, t]\right\}$ is $\gamma$-bounded and $\gamma\left(\mathscr{T}_{\alpha, t}\right) \leq C t^{\alpha}$, where $C$ depends on A only through its sectoriality constants.

Concerning $F$ and $G$, we shall assume:

(F) The function $F:[0, T] \times \Omega \times E \rightarrow E$ is Lipschitz continuous and of linear growth in its third variable, uniformly in $[0, T] \times \Omega$, i.e., there exist constants $L_{F}$ and $C_{F}$ such that for all $t \in[0, T], \omega \in \Omega$ and $x, y \in E$, we have

$$
\begin{aligned}
\|F(t, \omega, x)-F(t, \omega, y)\| & \leq L_{F}\|x-y\| \\
\|F(t, \omega, x)\| & \leq C_{F}(1+\|x\|) .
\end{aligned}
$$

Furthermore, for all $x \in E$, the map $(t, \omega) \mapsto F(t, \omega, x)$ is strongly measurable and adapted.

(G) The function $G:[0, T] \times \Omega \times E \rightarrow \mathscr{L}(H, E)$ is $\gamma$-Lipschitz continuous and of linear growth, uniformly in $[0, T] \times \Omega$, i.e., there exist constants $L_{G}$ and $C_{G}$ such that for all finite Borel measures $\mu$ on $[0, T]$, for all $\omega \in \Omega$ and for all $\phi_{1}, \phi_{2}, \phi \in L^{2}((0, T), \mu ; E) \cap \gamma\left(L^{2}((0, T), \mu), E\right)=: L_{\gamma}^{2}((0, T), \mu ; E)$, we have

$$
\begin{aligned}
\left\|G\left(\cdot, \omega, \phi_{1}\right)-G\left(\cdot, \omega, \phi_{2}\right)\right\|_{\gamma\left(L^{2}((0, T), \mu ; H), E\right)} & \leq L_{G}\left\|\phi_{1}-\phi_{2}\right\|_{L_{\gamma}^{2}((0, T), \mu ; E)} \\
\|G(\cdot, \omega, \phi)\|_{\gamma\left(L^{2}((0, T), \mu ; H), E\right)} & \leq C_{G}\left(1+\|\phi\|_{L_{\gamma}^{2}((0, T), \mu ; E)}\right) .
\end{aligned}
$$


Furthermore, for all $x \in E$ and $h \in H$, the map $(t, \omega) \mapsto G(t, \omega, x) h$ is strongly measurable and adapted.

The notion of $\gamma$-Lipschitz continuity has been introduced in [26], where various characterizations and examples were given. In particular, if $E$ is a type 2 Banach space (e.g. an $L^{p}$-space with $2 \leq p<\infty$ ), then every Lipschitz continuous function with values in $\gamma(H, E)$ is $\gamma$-Lipschitz continuous.

REMARK 3.2. It is implicit in condition $(\mathrm{G})$ that for all $\omega \in \Omega$ the functions $t \mapsto G(t, \omega, \phi(t))$ should represent an operator from $L^{2}((0, T), \mu ; H)$ to $E$. Note that the strong measurability in $H$ of $t \mapsto G^{*}(t, \omega, \phi(t)) x^{*}$ can be assumed without loss of generality. Indeed, the weak measurability of this functions is clear from the assumptions, and as explained in [25], there is no loss of generality in assuming that $H$ be separable; strong measurability then follows from the Pettis measurability theorem.

REMARK 3.3. In the present context, where the driving process is a cylindrical Brownian motion, it is not necessary to assume completeness of the filtration and/or progressive measurability of $F$ and $G$ (cf. [27, Proposition 2.10]).

REMARK 3.4. Taking $\phi=\mathbb{1} \otimes x$, we see that condition $(\mathrm{G})$ implies that $t \mapsto$ $G(t, \omega, x)$ defines an element in $\gamma\left(L^{2}((0, T), \mu ; H), E\right)$ for all $\omega \in \Omega$ and $x \in E$.

A mild solution of the problem (SCP) is a continuous adapted process $X:[0, T] \times$ $\Omega \rightarrow E$ such that

(1) for all $t \in[0, T], s \mapsto S(t-s) F(s, X(s))$ is strongly measurable and belongs to $L^{1}((0, t) ; E)$ almost surely;

(2) for all $t \in[0, T], s \mapsto S(t-s) G(s, X(s))$ is strongly measurable and stochastically integrable with respect to $W_{H}$;

(3) for all $t \in[0, T]$ we have, almost surely,

$$
X(t)=S(t) \xi+\mathbf{S} * F(\cdot, X)+\mathbf{S} \diamond G(\cdot, X) .
$$

Here, we used the notation

$$
\mathbf{S} * f(t):=\int_{0}^{t} S(t-s) f(s) \mathrm{d} s
$$

and

$$
\mathbf{S} \diamond \Phi(t):=\int_{0}^{t} S(t-s) \Phi(s) \mathrm{d} W_{H}(s)
$$

for deterministic and stochastic convolutions, respectively.

We recall that for $0 \leq a<b \leq T$ and $\mathscr{F}_{a}$-measurable sets $A \subseteq \Omega$, the stochastic integral of the indicator process $(t, \omega) \mapsto \mathbb{1}_{(a, b] \times A}(t, \omega) h \otimes x$ with respect to $W_{H}$ is defined as

$$
\int_{0}^{T} \mathbb{1}_{(a, b] \times A} \otimes[h \otimes x] \mathrm{d} W_{H}:=\mathbb{1}_{A} W_{H}\left(\mathbb{1}_{(a, b]} \otimes h\right) \otimes x .
$$


This definition extends to finite linear combinations of adapted indicator processes of the above form. For such processes $\Phi$, we have the following two-sided estimate.

PROPOSITION 3.5. [27, Theorem 5.9] Let E be a UMD Banach space and let $1<p<\infty$ be fixed. Then

$$
\mathbb{E}\left\|\int_{0}^{T} \Phi d W_{H}\right\|^{p} \approx \mathbb{E}\|\Phi\|_{\gamma\left(L^{2}(0, T ; H), E\right)}^{p},
$$

with implied constants depending only on $p$ and $E$.

By a density argument, this 'Itô isomorphism' extends to the Banach space $L_{\mathbb{F}}^{p}\left(\Omega ; \gamma\left(L^{2}(0, T ; H), E\right)\right)$ of all $\mathbb{F}$-adapted processes in $L^{p}\left(\Omega ; \gamma\left(L^{2}(0, T ; H), E\right)\right)$.

Existence and uniqueness of mild solutions in suitable Banach spaces of continuous adapted $E$-valued processes is proved by a fixed point argument. Following the approach of [26], for $0 \leq a<b<\infty, 1 \leq p<\infty$ and $0 \leq \alpha<\frac{1}{2}$, we denote by $V_{\alpha}^{p}([a, b] \times \Omega ; E)$ the Banach space of all continuous adapted processes $\phi:[a, b] \times \Omega \rightarrow E$ for which

$$
\|\phi\|_{\alpha, p}^{p}:=\mathbb{E}\|\phi\|_{C([a, b] ; E)}^{p}+\int_{a}^{b} \mathbb{E}\left\|s \mapsto(t-s)^{-\alpha} \phi(s)\right\|_{\gamma\left(L^{2}(a, t), E\right)}^{p} \mathrm{~d} t
$$

is finite, identifying processes that are indistinguishable.

We will need the following lemma, which allows us to estimate $\|\cdot\|_{\alpha, p}$-norms in terms of $\|\cdot\|_{\alpha, p}$-norms on smaller intervals.

LEMMA 3.6. Let $0 \leq a<b<c<d$ and $\phi:[a, d] \times \Omega \rightarrow E$ be an adapted process with $\phi \in V_{\alpha}^{p}([a, c] \times \Omega ; E) \cap V_{\alpha}^{p}([b, d] \times \Omega ; E)$. Then $\phi \in V_{\alpha}^{p}([a, d] \times \Omega ; E)$ and

$$
\|\phi\|_{V_{\alpha}^{p}([a, d] \times \Omega ; E)} \lesssim\|\phi\|_{V_{\alpha}^{p}([a, c] \times \Omega ; E)}+\|\phi\|_{V_{\alpha}^{p}([b, d] \times \Omega ; E)} .
$$

Proof. Clearly, $\phi$ belongs to $L^{p}(\Omega ; C([a, d] ; E))$ with

$$
\|\phi\|_{L^{p}(\Omega ; C([a, d] ; E))} \leq\|\phi\|_{L^{p}(\Omega ; C([a, c] ; E))}+\|\phi\|_{L^{p}(\Omega ; C([b, d] ; E))} .
$$

Concerning the second part of the $V_{\alpha}^{p}$-norm, we have

$$
\begin{aligned}
& \int_{a}^{b} \mathbb{E}\left\|s \mapsto(t-s)^{-\alpha} \phi(s)\right\|_{\gamma\left(L^{2}(a, t), E\right)}^{p} \mathrm{~d} t \\
& \leq \int_{a}^{c} \mathbb{E}\left\|s \mapsto(t-s)^{-\alpha} \phi(s)\right\|_{\gamma\left(L^{2}(a, t), E\right)}^{p} \mathrm{~d} t \\
& \quad+\int_{c}^{d} \mathbb{E}\left\|s \mapsto(t-s)^{-\alpha} \phi(s)\right\|_{\gamma\left(L^{2}(a, t), E\right)}^{p} \mathrm{~d} t .
\end{aligned}
$$

Now

$$
\int_{a}^{c} \mathbb{E}\left\|s \mapsto(t-s)^{-\alpha} \phi(s)\right\|_{\gamma\left(L^{2}(a, t), E\right)}^{p} \mathrm{~d} t \leq\|\phi\|_{V_{\alpha}^{p}([a, c] \times \Omega ; E)}^{p}
$$


and

$$
\begin{aligned}
& \left(\int_{c}^{d} \mathbb{E}\left\|s \mapsto(t-s)^{-\alpha} \phi(s)\right\|_{\gamma\left(L^{2}(a, t), E\right)}^{p} \mathrm{~d} t\right)^{\frac{1}{p}} \\
& \leq\left(\int_{c}^{d} \mathbb{E}\left\|s \mapsto(t-s)^{-\alpha} \phi(s)\right\|_{\gamma\left(L^{2}(a, b), E\right)}^{p} \mathrm{~d} t\right)^{\frac{1}{p}} \\
& \quad+\left(\int_{c}^{d} \mathbb{E}\left\|s \mapsto(t-s)^{-\alpha} \phi(s)\right\|_{\gamma\left(L^{2}(b, t), E\right)}^{p} \mathrm{~d} t\right)^{\frac{1}{p}} \\
& \leq(c-b)^{-\alpha}(d-c) \mathbb{E}\|\phi\|_{\gamma\left(L^{2}(a, b), E\right)}+\|\phi\|_{V_{\alpha}^{p}([b, d] \times \Omega ; E)} .
\end{aligned}
$$

The inequality of the first terms in the last step follows from the right ideal property for spaces of $\gamma$-radonifying operators. Now, observe that

$$
\begin{aligned}
\mathbb{E}\|\phi\|_{\gamma\left(L^{2}(a, b), E\right)}^{p} & =\frac{1}{c-b} \int_{b}^{c} \mathbb{E}\|\phi\|_{\gamma\left(L^{2}(a, b), E\right)}^{p} \mathrm{~d} t \leq \frac{1}{c-b} \int_{b}^{c} \mathbb{E}\|\phi\|_{\gamma\left(L^{2}(a, t), E\right)}^{p} \mathrm{~d} t \\
& \leq \frac{c^{\alpha p}}{c-b} \int_{b}^{c} \mathbb{E}\left\|s \mapsto(t-s)^{-\alpha} \phi(s)\right\|_{\gamma\left(L^{2}(a, t), E\right)}^{p} \mathrm{~d} t \\
& \leq \frac{c^{\alpha p}}{c-b}\|\phi\|_{V_{\alpha}^{p}([a, c] \times \Omega ; E)} .
\end{aligned}
$$

Here, we have used covariance domination. Collecting the estimates, the claim follows.

THEOREM 3.7. (Existence and uniqueness, [26, Proposition 6.1]) Let E be a UMD space, and suppose that assumptions $(\mathrm{A}),(\mathrm{F})$ and $(\mathrm{G})$ are satisfied. Fix $2<p<\infty$ and $\frac{1}{p}<\alpha<\frac{1}{2}$ and let $\xi \in L^{p}\left(\Omega, \mathscr{F}_{0} ; E\right)$ be given. The mapping

$$
\Lambda_{\xi, T}: \phi \mapsto S(\cdot) \xi+\mathbf{S} * F(\cdot, \phi)+\mathbf{S} \diamond G(\cdot, \phi)
$$

defines a Lipschitz continuous mapping on the space $V_{\alpha}^{p}([0, T] \times \Omega ; E)$. Its Lipschitz constant is independent of $\xi$ and depends on $A, F, G$ only through the constants $L_{F}, L_{G}$, and the sectoriality constants of $A$ and tends toward 0 as $T \downarrow 0$.

For small $T_{0}>0$, the mapping $\Lambda_{\xi, T_{0}}$ has a unique fixed point in $V_{\alpha}^{p}\left(\left[0, T_{0}\right] \times \Omega ; E\right)$, and this fixed point turns out to be a mild solution of (SCP) on the interval $[0, T]$. Repeating this argument inductively in conjunction with Lemma 3.6, one obtains a unique solution $X$ in $V_{\alpha}^{p}([0, T] \times \Omega ; E)$ of $(\mathrm{SCP})$ on the interval $[0, T]$ (see [26, Theorem 6.2]).

We note that for $1 \leq q \leq p<\infty$ and $0 \leq \alpha<\frac{1}{2}$, we have a continuous embedding

$$
V_{\alpha}^{p}([0, T] \times \Omega ; E) \hookrightarrow V_{\alpha}^{q}([0, T] \times \Omega ; E) .
$$

Furthermore, for $1 \leq p<\infty$ and $0 \leq \alpha<\beta<\frac{1}{2}$, the ideal property yields a continuous embedding

$$
V_{\beta}^{p}([0, T] \times \Omega ; E) \hookrightarrow V_{\alpha}^{p}([0, T] \times \Omega ; E) .
$$


These embeddings imply consistency of solutions for different values of $\alpha$ and $p$.

The next lemma provides a way to test whether a given process belongs to $V_{\alpha}^{p}([0, T] \times \Omega ; E)$. By $L_{\mathbb{F}}^{p}\left(\Omega ; C^{\lambda}([0, T] ; E)\right)$, we denote the Banach space of all continuous adapted processes $\phi:[0, T] \times \Omega \rightarrow E$ belonging to $L^{p}\left(\Omega ; C^{\lambda}([0, T] ; E)\right)$, once more identifying processes that are indistinguishable.

LEMMA 3.8. Let $2<p<\infty$ and $\frac{1}{p}<\alpha<\frac{1}{2}$, and let $E$ be a Banach space with type $\tau \in[1,2)$. Then for $\lambda>\frac{1}{\tau}-\frac{1}{2}$, we have a continuous embedding

$$
L_{\mathbb{F}}^{p}\left(\Omega ; C^{\lambda}([0, T] ; E)\right) \hookrightarrow V_{\alpha}^{p}([0, T] \times \Omega ; E) .
$$

Proof. Pick $q>0$ such that $\alpha<\frac{1}{2}-\frac{1}{q}$. By [26, Lemma 3.3] and the fact that $C^{\lambda}([0, T] ; E)$ embeds into the Besov space $B_{q, \tau}^{\frac{1}{\tau}-\frac{1}{2}}(0, T ; E)$, for all $f \in C^{\lambda}([0, T] ; E)$ we have

$$
\sup _{t \in(0, T)}\left\|s \mapsto(t-s)^{-\alpha} f(s)\right\|_{\gamma\left(L^{2}(0, t), E\right)}^{p} \lesssim\|f\|_{B_{q, \tau}^{\frac{1}{\tau}-\frac{1}{2}}(0, T ; E)} \lesssim\|f\|_{C^{\lambda}([0, T] ; E)} .
$$

Thus, considering adapted $\phi \in L^{p}\left(\Omega ; C^{\lambda}([0, T] ; E)\right)$, we see that

$$
\int_{0}^{T} \mathbb{E}\left\|s \mapsto(t-s)^{-\alpha} \phi(s)\right\|_{\gamma\left(L^{2}(0, t), E\right)}^{p} \mathrm{~d} t \lesssim T\|\phi\|_{L^{p}\left(\Omega ; C^{\lambda}([0, T] ; E)\right)}^{p} .
$$

The other part of the norm of $V_{\alpha}^{p}([0, T] \times \Omega ; E)$ can clearly be estimated by the norm of $L^{p}\left(\Omega ; C^{\lambda}([0, T] ; E)\right.$.

\section{Continuous dependence on the coefficients}

We now take up our main line of study and approximate simultaneously the coefficients $A, F, G$ and the initial datum $\xi$ in equation (SCP). Regarding the approximation of $A$, we make the following assumptions:

(A1) The operators $A$ and $A_{n}$ are densely defined, closed, and uniformly sectorial on $E$ in the sense, there exist numbers $M \geq 1$ and $w \in \mathbb{R}$ such that $A$ and each $A_{n}$ is sectorial of type $(M, w)$.

(A2) The operators $A_{n}$ converge to $A$ in the strong resolvent sense:

$$
\lim _{n \rightarrow \infty} R\left(\lambda, A_{n}\right) x=R(\lambda, A) x
$$

for all $\operatorname{Re} \lambda>w$ and $x \in E$.

Under (A1), the operators $A$ and $A_{n}$ generate strongly continuous analytic semigroups $\mathbf{S}, \mathbf{S}_{n}$ satisfying the uniform bounds

$$
\begin{aligned}
\|S(t)\|,\left\|S_{n}(t)\right\| & \leq M e^{w t}, \quad t \geq 0, \\
\|A S(t)\|,\left\|A_{n} S_{n}(t)\right\| & \leq \frac{M^{\prime}}{t} e^{w t}, \quad t>0 .
\end{aligned}
$$

The following Trotter-Kato type approximation theorem is well known; see [2, Theorem 3.6.1] for part (1), part (2) follows from a contour integral argument. 
LEMMA 4.1. Assume (A1) and (A2).

(1) For all $t \in[0, \infty)$ and $x \in E$, we have $S_{n}(t) x \rightarrow S(t) x$, and the convergence is uniform on compact subsets of $[0, \infty) \times E$.

(2) For all $t \in(0, \infty)$ and $x \in E$, we have $A_{n} S_{n}(t) x \rightarrow A S(t) x$, and the convergence is uniform on compact subsets of $(0, \infty) \times E$.

As a consequence, we see that under (A1) and (A2), and for each $\beta \in[0,1)$, the functions $M_{n}^{\beta}(t):=t^{\beta} S_{n}(t)$ and $M^{\beta}(t):=t^{\beta} S(t)$ satisfy the hypotheses of Lemma 2.6. Indeed, condition 2.6(1) is clear, condition 2.6(2) follows from Lemma 4.1, and condition 2.6(3) follows from Lemma 3.1 according to which the sets

$$
\left\{s^{\beta} S_{n}(s): s \in(0, t)\right\}
$$

are $\gamma$-bounded in $\mathscr{L}(E)$ with a uniform $\gamma$-bound of order $t^{\beta}$.

We will make the following assumptions on the nonlinearities $F$ and $F_{n}$ :

(F1) The maps $F, F_{n}:[0, T] \times \Omega \times E \rightarrow E$ are uniformly Lipschitz continuous and of linear growth in the sense that they satisfy (F) with uniform Lipschitz and growth constants. Furthermore, $F$ and $F_{n}$ satisfy the measurability assumption in $(\mathrm{F})$.

(F2) For almost all $(t, \omega) \in[0, T] \times \Omega$, we have $F_{n}(t, \omega, x) \rightarrow F(t, \omega, x)$ in $E$ for all $x \in E$.

Similar assumptions are made on $G$ and $G_{n}$ :

(G1) The maps $G, G_{n}:[0, T] \times \Omega \times E \rightarrow \gamma(H, E)$ are uniformly $\gamma$-Lipschitz continuous and of linear growth in the sense that they satisfy $(\mathrm{G})$ with uniform $\gamma$-Lipschitz and growth constants. Furthermore, $G$ and $G_{n}$ satisfies the measurability assumption in $(\mathrm{G})$.

(G2) For almost all $(t, \omega) \in[0, T] \times \Omega$, we have $G_{n}(\cdot, \omega, x) \rightarrow G(\cdot, \omega, x)$ in $\gamma\left(L^{2}(0, T, \mu ; H), E\right)$, for all $x \in E$ and all finite measures $\mu$ on $[0, T]$.

We will need a lemma on convergence of random variables with values in spaces of Hölder continuous functions. For $\eta \in L^{p}\left(\Omega ; C^{\lambda}([0, T] ; E)\right)$, we denote by $\eta_{t} \in$ $L^{p}(\Omega)$ the random variable $\left(\eta_{t}\right)(\omega):=(\eta(\omega))(t)$.

LEMMA 4.2. Let $E$ be a Banach space, let $1<p<\infty$ and $\lambda>0$, and suppose that $\eta_{n}, \eta \in L^{p}\left(\Omega ; C^{\lambda}([0, T] ; E)\right)$ satisfy

(1) $\sup _{n}\left\|\eta_{n}\right\|_{L^{p}\left(\Omega ; C^{\lambda}([0, T] ; E)\right)}<\infty$;

(2) $\left(\eta_{n}\right)_{t} \rightarrow \eta_{t}$ in measure for all $t \in[0, T]$;

Then, $\eta_{n} \rightarrow \eta$ in $L^{q}\left(\Omega ; C^{\mu}([0, T] ; E)\right)$ for all $1 \leq q<p$ and $0 \leq \mu<\lambda$.

Proof. We fix $0<\mu<\lambda$ and put $\zeta_{n}:=\eta_{n}-\eta$.

Let $M:=\sup _{n}\left\|\zeta_{n}\right\|_{L^{p}\left(\Omega ; C^{\lambda}([0, T] ; E)\right)}^{p}$. Chebyshev's inequality implies that

$$
\sup _{n} \mathbb{P}\left(\left\|\zeta_{n}\right\|_{C^{\lambda}([0, T] ; E)} \geq R\right) \leq R^{-p} M \rightarrow 0 \text { as } R \rightarrow \infty .
$$

It follows from this and assumption (2) that $\zeta_{n} \rightarrow 0$ in measure in $C^{\mu}([0, T] ; E)$. The proof is the same as that of [24, Proposition 2.1] where the finite dimensional 
situation was considered. See also [3, Lemma A.1] for further convergence results of this form.

As a consequence of the boundedness of $\left(\zeta_{n}\right)$ in $L^{p}\left(\Omega ; C^{\lambda}([0, T] ; E)\right)$, the random variables $\left\|\zeta_{n}\right\|_{C^{\lambda}([0, T] ; E)}^{q}$-and hence also the random variables $\left\|\zeta_{n}\right\|_{C^{\mu}([0, T] ; E)}^{q}$-are uniformly integrable for all $1 \leq q<p$. It follows from [17, Proposition 4.12] that $\zeta_{n} \rightarrow 0$ in $L^{q}\left(\Omega ; C^{\mu}([0, T] ; E)\right)$.

We are now in a position to state and prove the main abstract result of this paper. In its formulation, we use that UMD Banach spaces have nontrivial type. In fact, UMD Banach spaces are super-reflexive, super-reflexive spaces are $K$-convex, and $K$-convexity is equivalent to having nontrivial type. For more details and references to the literature, we refer to $[15,16]$.

In what follows, we will consider $0 \leq \alpha<\frac{1}{2}$ and $2<p<\infty$ to be fixed and write

$$
X=\operatorname{sol}(A, F, G, \xi)
$$

to indicate that $X$ is the unique mild solution of $(\mathrm{SCP})$ in $V_{\alpha}^{p}([0, T] \times \Omega ; E)$ with coefficients $(A, F, G)$ and initial datum $\xi$.

THEOREM 4.3. Let $E$ be a UMD Banach space, let $\tau \in(1,2]$ be its type, and suppose that $\frac{1}{p}<1-\frac{1}{\tau}$. Suppose further that the operators $A$ and $A_{n}$ satisfy (A1) and (A2), the nonlinearities $F$ and $F_{n}$ satisfy (F1) and (F2), the nonlinearities $G$ and $G_{n}$ satisfy (G1) and (G2), and the initial data $\xi$ and $\xi_{n}$ satisfy $\xi_{n} \rightarrow \xi$ in $L^{p}\left(\Omega, \mathscr{F}_{0} ; E\right)$. Then, whenever $\frac{1}{\tau}-\frac{1}{2}+\frac{1}{p}<\alpha<\frac{1}{2}$, the mild solutions

$$
X:=\operatorname{sol}(A, F, G, \xi), \quad X_{n}:=\operatorname{sol}\left(A_{n}, F_{n}, G_{n}, \xi_{n}\right)
$$

satisfy

$$
X_{n} \rightarrow X \text { in } V_{\alpha}^{q}([0, T] \times \Omega ; E)
$$

for all $1 \leq q<p$. In particular, $X_{n} \rightarrow X$ in $L^{q}(\Omega ; C([0, T] ; E))$ for all $1 \leq q<p$.

We structure the proof through a series of lemmas.

LEMMA 4.4. Let $1 \leq p<\infty$ and $0 \leq \alpha<\frac{1}{2}$. Suppose that the operators $A_{n}$ and A satisfy (A1) and (A2) and that $\xi_{n} \rightarrow \xi$ in $L^{p}\left(\Omega, \mathscr{F}_{0} ; E\right)$. Then,

$$
S_{n}(\cdot) \xi_{n} \rightarrow S(\cdot) \xi \quad \text { in } V_{\alpha}^{p}([0, T] \times \Omega ; E)
$$

Proof. By Lemma 4.1(1), for every $x \in E$, we have $S_{n}(t) x \rightarrow S(t) x$ in $C([0, T] ; E)$ as $n \rightarrow \infty$. Hence, $S_{n}(\cdot) \xi \rightarrow S(\cdot) \xi$ in $C([0, T] ; E)$ almost surely, and noting that the semigroups $\mathbf{S}_{n}$ are uniformly bounded on $[0, T]$, say by a constant $M_{T}$, we infer from dominated convergence that

$$
\left\|S_{n}(\cdot) \xi-S(\cdot) \xi\right\|_{L^{p}(\Omega ; C([0, T] ; E))} \rightarrow 0
$$


Also, $\left\|S_{n}(t) \xi_{n}-S_{n}(t) \xi\right\| \leq M_{T}\left\|\xi_{n}-\xi\right\|$, which implies

$$
\mathbb{E}\left\|S_{n}(\cdot) \xi_{n}-S_{n}(\cdot) \xi\right\|_{C([0, T] ; E)}^{p} \leq M_{T}\left\|\xi_{n}-\xi\right\|_{L^{p}(\Omega ; E)}^{p} \rightarrow 0 .
$$

Combining these estimates, we obtain

$$
\mathbb{E}\left\|S_{n}(\cdot) \xi_{n}-S(\cdot) \xi\right\|_{C([0, T] ; E)}^{p} \rightarrow 0 .
$$

Choose $\beta>0$ such that $\alpha+\beta<\frac{1}{2}$ and put $M_{n}(t):=t^{\beta} S_{n}(t)$ and $M(t):=t^{\beta} S(t)$. The finite Borel measures $\mu_{t}^{\alpha}$ on $(0, t)$ are defined by

$$
\mu_{t}^{\alpha}(A)=\int_{A}(t-s)^{-2 \alpha} \mathrm{d} s .
$$

It is straightforward (see [26]) to verify that

$$
\phi \in \gamma\left(L^{2}\left(0, t, \mu_{t}^{\alpha}\right) ; E\right) \Longleftrightarrow\left[s \mapsto(t-s)^{-\alpha} \phi(s)\right] \in \gamma\left(L^{2}(0, t), E\right)
$$

with identical norms. Almost surely, we have

$$
\begin{aligned}
& \left\|S_{n}(\cdot) \xi-S(\cdot) \xi\right\|_{\gamma\left(L^{2}\left(0, t, \mu_{t}^{\alpha}\right), E\right)} \\
& \quad=\left\|s \mapsto(t-s)^{-\alpha} s^{-\beta}\left(M_{n}(s) \xi-M(s) \xi\right)\right\|_{\gamma\left(L^{2}(0, t), E\right)} .
\end{aligned}
$$

Let $\gamma_{T}:=\sup _{n} \gamma\left(\left\{t^{\beta} S_{n}(t): 0 \leq t \leq T\right\}\right)$. By Lemma 3.1 and assumption (A1), $\gamma_{T}<\infty$. Using the observation (4.3) combined with Proposition 2.5 and Lemma 2.1, almost surely we obtain, for all $t \in(0, T)$ and indices $n$,

$$
\begin{aligned}
\left\|S_{n}(\cdot) \xi\right\|_{\gamma\left(L^{2}\left(0, t, \mu_{t}^{\alpha}\right), E\right)} & \leq \gamma_{T}\left\|s \mapsto(t-s)^{-\alpha} s^{-\beta} \xi\right\|_{\gamma\left(L^{2}(0, t), E\right)} \\
& =\gamma_{T}\left\|s \mapsto(t-s)^{-\alpha} s^{-\beta}\right\|_{L^{2}(0, t)}\|\xi\| .
\end{aligned}
$$

The same estimate also holds with $\mathbf{S}_{n}$ replaced with $\mathbf{S}$. Note that

$$
\left\|s \mapsto(t-s)^{-\alpha} s^{-\beta}\right\|_{L^{2}(0, t)}^{2}=t^{1-2 \alpha-2 \beta} \int_{0}^{1} r^{-2 \beta}(1-r)^{-2 \alpha} \mathrm{d} r,
$$

which is finite. Since $\alpha+\beta<\frac{1}{2}$, the supremum over $t \in[0, T]$ of this expression is bounded, say by $C_{T, \alpha, \beta}$. Hence, we have

$$
\mathbb{E}\left\|S_{n}(\cdot) \xi-S(\cdot) \xi\right\|_{\gamma\left(L^{2}\left(0, t, \mu_{t}^{\alpha}\right), E\right)}^{p} \leq 2 \gamma_{T}^{p} C_{T, \alpha, \beta}^{p}\|\xi\|_{L^{p}(\Omega ; E)}^{p} .
$$

Furthermore, by the observation following Lemma 4.1, we may apply Lemma 2.6 to the functions $M_{n}, M$ and the $\gamma$-radonifying operators represented by the functions $s \mapsto(t-s)^{-\alpha} s^{-\beta} \xi(\omega)$ to conclude that

$$
\left\|S_{n}(\cdot) \xi-S(\cdot) \xi\right\|_{\gamma\left(L^{2}\left(0, t, \mu_{t}^{\alpha}\right), E\right)} \rightarrow 0
$$

almost surely. 
Hence, by dominated convergence,

$$
\int_{0}^{T} \mathbb{E}\left\|S_{n}(\cdot) \xi-S(\cdot) \xi\right\|_{\gamma\left(L^{2}\left(0, t, \mu_{t}^{\alpha}\right), E\right)}^{p} \mathrm{~d} t \rightarrow 0 .
$$

Together with (4.1), this shows that $S_{n}(\cdot) \xi \rightarrow S(\cdot) \xi$ in $V_{\alpha}^{p}([0, T] \times \Omega ; E)$.

Arguing as before, we see that

$$
\left\|S_{n}(\cdot) \xi_{n}-S_{n}(\cdot) \xi\right\|_{\gamma\left(L^{2}\left(0, t, \mu_{t}^{\alpha}\right), E\right)} \leq \gamma_{T}\left\|s \mapsto(t-s)^{-\alpha} s^{-\beta}\right\|_{L^{2}(0, t)}\left\|\xi_{n}-\xi\right\|,
$$

so

$$
\int_{0}^{T} \mathbb{E}\left\|S_{n}(\cdot) \xi_{n}-S_{n}(\cdot) \xi\right\|_{\gamma\left(L^{2}\left(0, t, \mu_{t}^{\alpha}\right), E\right)}^{p} \mathrm{~d} t \lesssim\left\|\xi_{n}-\xi\right\|_{L^{p}(\Omega ; E)}^{p} \rightarrow 0 .
$$

Combining this with (4.2), this gives $\left\|S_{n}(\cdot) \xi_{n}-S_{n}(\cdot) \xi\right\| \rightarrow 0$ in $V_{\alpha}^{p}([0, T] \times \Omega ; E)$.

Collecting the estimates, the proof is complete.

LEMMA 4.5. Let $E$ be a UMD Banach space and assume (A1), (A2), (F1), (F2), (G1), and (G2). Suppose that $\frac{1}{p}<\alpha<\frac{1}{2}$ and let $\phi \in V_{\alpha}^{p}([0, T] \times \Omega ; E)$ be given. Then, for all $0 \leq \lambda<\alpha-\frac{1}{p}$ and $1 \leq q<p$ we have

(1) $\mathbf{S}_{n} * F_{n}(\cdot, \phi)-\mathbf{S}_{n} * F(\cdot, \phi) \rightarrow 0$ in $L^{p}\left(\Omega ; C^{\lambda}([0, T] ; E)\right)$;

(2) $\mathbf{S}_{n} \diamond G_{n}(\cdot, \phi)-\mathbf{S}_{n} \diamond G(\cdot, \phi) \rightarrow 0$ in $L^{p}\left(\Omega ; C^{\lambda}([0, T] ; E)\right)$;

(3) $\mathbf{S}_{n} * F(\cdot, \phi) \rightarrow \mathbf{S} * F(\cdot, \phi)$ in $L^{q}\left(\Omega ; C^{\lambda}([0, T] ; E)\right)$;

(4) $\mathbf{S}_{n} \diamond G(\cdot, \phi) \rightarrow \mathbf{S} \diamond G(\cdot, \phi)$ in $L^{q}\left(\Omega ; C^{\lambda}([0, T] ; E)\right)$.

Proof. (1) Let us denote the fractional convolution operator of exponent $0<a<1$ associated with $A_{n}$ by $I_{a, n}$ :

$$
I_{a, n} f(t):=\frac{1}{\Gamma(a)} \int_{0}^{t}(t-s)^{a-1} S_{n}(t-s) f(s) \mathrm{d} s .
$$

Pick any $r>2$ and noting that $\frac{1}{r}+\lambda<1$, $a$ such that $\frac{1}{r}+\lambda<a<1$. Then,

$$
\mathbf{S}_{n} * f=I_{a, n} I_{1-a, n} f
$$

for all $f \in L^{r}(0, T ; E)$. Moreover, by the uniform sectoriality of the operators $A_{n}$, the operators $I_{a, n}$ are uniformly bounded on $L^{r}(0, T ; E)$ and uniformly bounded from $L^{r}(0, T ; E)$ to $C^{\lambda}([0, T] ; E)$ (cf. $\left.[8,9]\right)$. Hence,

$$
\begin{aligned}
\mathbb{E}\left\|\mathbf{S}_{n} * F_{n}(\cdot, \phi)-\mathbf{S}_{n} * F(\cdot, \phi)\right\|_{C^{\lambda}([0, T] ; E)}^{p} & \lesssim \mathbb{E}\left\|I_{1-a, n}\left(F_{n}(\cdot, \phi)-F(\cdot, \phi)\right)\right\|_{L^{r}(0, T ; E)}^{p} \\
& \left.\lesssim \mathbb{E} \| F_{n}(\cdot, \phi)-F(\cdot, \phi)\right) \|_{L^{r}(0, T ; E)}^{p},
\end{aligned}
$$

Now, note that

$$
\left\|F_{n}(t, \omega, \phi(t, \omega))-F(t, \omega, \phi(t, \omega))\right\| \leq 2 C_{F}(1+\|\phi(t, \omega)\|),
$$

and the right-hand side belongs to $L^{p}\left(\Omega ; L^{r}(0, T ; E)\right)$. Since for almost all $(t, \omega)$, we have $F_{n}(t, \omega, \phi(t, \omega)) \rightarrow F(t, \omega, \phi(t, \omega)), \mathbb{E}\left\|F_{n}(\cdot, \phi)-F(\cdot, \phi)\right\|_{L^{r}(0, T ; E)}^{p} \rightarrow 0$ follows by dominated convergence. Thus, $\mathbb{E}\left\|\mathbf{S}_{n} * F_{n}(\cdot, \phi)-\mathbf{S}_{n} * F(\cdot, \phi)\right\|_{C^{\lambda}([0, T] ; E)}^{p} \rightarrow$ 0 as $n \rightarrow \infty$. 
(2) By [26, Proposition 4.2],

$$
\begin{aligned}
& \left\|\mathbf{S}_{n} \diamond G_{n}(\cdot, \phi)-\mathbf{S}_{n} \diamond G(\cdot, \phi)\right\|_{L^{p}\left(\Omega ; C^{\lambda}([0, T] ; E)\right)} \\
& \quad \lesssim\left(\int_{0}^{T} \mathbb{E}\left\|G_{n}(\cdot, \phi)-G(\cdot, \phi)\right\|_{\gamma\left(L^{2}\left(0, t, \mu_{t}^{\alpha} ; H\right), E\right)}^{p} \mathrm{~d} t\right)^{\frac{1}{p}} .
\end{aligned}
$$

We note that for almost all $\omega$, we have

$$
\left\|G_{n}(\cdot, \omega, \phi)-G(\cdot, \omega, \phi)\right\|_{\gamma\left(L^{2}\left(0, t, \mu_{t}^{\alpha} ; H\right), E\right)} \leq C_{G}\left(1+\|\phi(\cdot, \omega)\|_{L_{\gamma}^{2}\left(0, t, \mu_{t}^{\alpha} ; E\right)}\right) .
$$

The right-hand side belongs to $L^{p}((0, T) \times \Omega)$ since $\phi \in V_{\alpha}^{p}([0, T] \times \Omega ; E)$. Hence, if we prove that $G_{n}(\cdot, \omega, \phi(\cdot, \omega)) \rightarrow G(\cdot, \omega, \phi(\cdot, \omega))$ in $\gamma\left(L^{2}\left(0, t, \mu_{t}^{\alpha} ; H\right), E\right)$ for almost all $t$ and $\omega$, then, by dominated convergence, we conclude that $\mathbf{S}_{n} \diamond G_{n}(\cdot, \phi)-$ $\mathbf{S} \diamond G(\cdot, \phi) \rightarrow 0$ in $L^{p}\left(\Omega ; C^{\lambda}([0, T] ; E)\right)$.

Fix $t \in[0, T]$ and $\omega \in \Omega$. For notational convenience, we shall suppress the dependence on $\omega$. Let $\psi:=\sum_{k=1}^{K} \mathbb{1}_{A_{k}} x_{k}$ be a simple function. Then

$$
G(\cdot, \psi(\cdot))=\sum_{k=1}^{K} \mathbb{1}_{A_{k}} G\left(\cdot, x_{k}\right)
$$

and similarly for $G_{n}$. Note that $G_{n}\left(\cdot, x_{k}\right) \rightarrow G\left(\cdot, x_{k}\right)$ in $\gamma\left(L^{2}\left(0, t, \mu_{t}^{\alpha} ; H\right), E\right)$ for all $1 \leq k \leq K$ by assumption (G2). Since multiplication by $\mathbb{1}_{A_{k}}$ is a bounded operator on $L^{2}\left(0, t, \mu_{t}^{\alpha} ; H\right)$, it follows from the ideal property of $\gamma$-radonifying operators that $\mathbb{1}_{A_{k}}(\cdot) G\left(\cdot, x_{k}\right) \rightarrow \mathbb{1}_{A_{k}}(\cdot) G\left(\cdot, x_{k}\right)$ in $\gamma\left(L^{2}\left(0, t, \mu_{t}^{\alpha} ; H\right), E\right)$ for all $1 \leq k \leq K$. Summing up it follows that $G_{n}(\cdot, \psi) \rightarrow G(\cdot, \psi)$ in $\gamma\left(L^{2}\left(0, t, \mu_{t}^{\alpha} ; H\right), E\right)$.

Now let $\psi, \psi_{0} \in \gamma\left(L^{2}\left(0, t ; \mu_{t}^{\alpha}\right), E\right)$ be arbitrary. By assumption (G1), we have

$$
\begin{aligned}
& \left\|G_{n}(\cdot, \psi)-G(\cdot, \psi)\right\|_{\gamma\left(L^{2}\left(0, t ; \mu_{t}^{\alpha} ; H\right), E\right)} \\
& \quad \leq 2 L_{G}\left\|\psi-\psi_{0}\right\|_{L_{\gamma}^{2}\left(0, t ; \mu_{t}^{\alpha} ; E\right)}+\left\|G_{n}\left(\cdot, \psi_{0}\right)-G\left(\cdot, \psi_{0}\right)\right\|_{\gamma\left(L^{2}\left(0, t, \mu_{t}^{\alpha} ; H\right), E\right)} .
\end{aligned}
$$

Thus, by first choosing a simple function $\psi_{0}$ close enough to $\psi$ and then $n$ large enough, we see that for any $\psi \in \gamma\left(L^{2}\left(0, t ; \mu_{t}^{\alpha}\right), E\right)$ we have $G_{n}(\cdot, \psi) \rightarrow G(\cdot, \psi)$ in $\gamma\left(L^{2}\left(0, t, \mu_{t}^{\alpha} ; H\right), E\right)$.

Using this result pathwise, it follows that, almost surely, $G_{n}(\cdot, \phi) \rightarrow G(\cdot, \phi)$ in $\gamma\left(L^{2}\left(0, t, \mu_{t}^{\alpha} ; H\right), E\right)$. Since $t$ was arbitrary, this finishes the proof of (2).

(3) We pick $\mu$ such that $\lambda<\mu<\alpha-\frac{1}{p}$. Arguing as in the proof of (1), for large $r$ we have

$$
\begin{aligned}
\mathbb{E}\left\|\mathbf{S}_{n} * F(\cdot, \phi)-\mathbf{S} * F(\cdot, \phi)\right\|_{C^{\mu}([0, T] ; E)}^{p} & \lesssim 2 \mathbb{E}\|F(\cdot, \phi)\|_{L^{r}(0, T ; E)}^{p} \\
& \lesssim \mathbb{E}\left(\int_{0}^{T}(1+\|\phi(t)\|)^{r} \mathrm{~d} t\right)^{\frac{p}{r}}<\infty
\end{aligned}
$$

Now observe that, almost surely, we have

$$
\int_{0}^{t} S_{n}(t-s) F(s, \phi(s)) \mathrm{d} t \rightarrow \int_{0}^{t} S(t-s) F(s, \phi(s)) \mathrm{d} s
$$


in $E$ for every $t \in[0, T]$, by dominated convergence. Applying the dominated convergence theorem second time, we see that $\left[\mathbf{S}_{n} * F(\cdot, \phi)\right](t) \rightarrow[\mathbf{S} * F(\cdot, \phi)](t)$ in $L^{p}(\Omega ; E)$. Convergence of the deterministic convolution in $L^{q}\left(\Omega ; C^{\lambda}([0, T] ; E)\right)$ follows from Lemma 4.2.

(4) Arguing similarly as in the proof of (3), we see that $\mathbf{S}_{n} \diamond G(\cdot, \phi)-\mathbf{S} \diamond G(\cdot, \phi)$ is bounded in $L^{p}\left(\Omega ; C^{\mu}([0, T] ; E)\right)$ for $\lambda<\mu<\alpha-\frac{1}{p}$.

Now fix $t \in[0, T]$. Since $s \mapsto(t-s)^{-\alpha} G(s, \phi(s))$ belongs to $\left.\gamma\left(L^{2}(0, t ; H), E\right)\right)$ almost surely, it follows from Lemma 2.6 as in the proof of Lemma 4.4 that, almost surely, $S_{n}(t-\cdot) G(\cdot, \phi(\cdot)) \rightarrow S(t-\cdot) G(\cdot, \phi(\cdot))$ in $\gamma\left(L^{2}(0, t ; H), E\right)$. Furthermore, by Proposition 2.5,

$$
\begin{aligned}
& \left\|S_{n}(t-\cdot) G(\cdot, \phi(\cdot))\right\|_{\gamma\left(L^{2}(0, t ; H), E\right)} \\
& \quad \leq \gamma_{\alpha, T}\left\|(t-\cdot)^{-\alpha} G(\cdot, \phi(\cdot))\right\|_{\gamma\left(L^{2}(0, t ; H), E\right)} \leq \gamma_{\alpha, T} C_{G}\left(1+\|\phi\|_{\alpha, p}\right),
\end{aligned}
$$

where $\gamma_{\alpha, T}:=\sup _{n} \gamma\left(\left\{s^{\alpha} S_{n}(s): 0<s \leq T\right\}\right)<\infty$ by Lemma 3.1 and the uniform sectoriality of the operators $A_{n}$. Hence, by dominated convergence and the Itô isomorphism,

$$
\begin{aligned}
& \mathbb{E}\left\|\left[S_{n} \diamond G(\cdot, \phi)\right](t)-[S \diamond G(\cdot, \phi)](t)\right\|^{p} \\
& \quad \approx \mathbb{E}\left\|S_{n}(t-\cdot) G(\cdot, \phi(\cdot))-S(t-\cdot) G(\cdot, \phi(\cdot))\right\|_{\gamma\left(L^{2}(0, t ; H), E\right)}^{p} \rightarrow 0 .
\end{aligned}
$$

Now, Lemma 4.2 yields $\mathbf{S}_{n} \diamond G(\phi) \rightarrow \mathbf{S} \diamond G(\phi)$ in $L^{q}\left(\Omega ; C^{\lambda}([0, T] ; E)\right)$.

Proof of Theorem 4.3. We may replace $q$ be some larger value and thereby assume that $q \in(2, p)$ and $\alpha \in\left(\frac{1}{q}, \frac{1}{2}\right)$.

Step 1 We prove the theorem for small $T_{0}$.

Let $\Lambda$ and $\Lambda_{n}$ denote the Lipschitz continuous mappings on $V_{\alpha}^{q}([0, T] \times \Omega ; E)$ used to solve (SCP) with data $(A, F, G, \xi)$ and $\left(A_{n}, F_{n}, G_{n}, \xi_{n}\right)$, respectively (see Theorem 3.7). We choose $T_{0}>0$ so small that, for some constant $0 \leq c<1$,

$$
\sup _{n}\left\|\Lambda_{n}(\phi)-\Lambda_{n}(\psi)\right\|_{\alpha, q} \leq c\|\phi-\psi\|_{\alpha, q}
$$

for all $\phi, \psi \in V_{\alpha}^{q}\left(\left[0, T_{0}\right] \times \Omega ; E\right)$. This is possible by Theorem 3.7, noting that all estimates involving $A_{n}, F_{n}, G_{n}$ are uniform in $n$.

We denote by $X$ and $X_{n}$ the unique fixed points in $V_{\alpha}^{p}\left(\left[0, T_{0}\right] \times \Omega ; E\right)$ of the operators $\Lambda$ and $\Lambda_{n}$, so that

$$
\begin{aligned}
X & =\Lambda(X)=S(\cdot) \xi+\mathbf{S} * F(\cdot, X)+\mathbf{S} \diamond G(\cdot, X), \\
X_{n} & =\Lambda\left(X_{n}\right)=S_{n}(\cdot) \xi_{n}+\mathbf{S}_{n} * F_{n}\left(\cdot, X_{n}\right)+\mathbf{S}_{n} \diamond G_{n}\left(\cdot, X_{n}\right) .
\end{aligned}
$$

We have

$$
\left\|X-X_{n}\right\|_{\alpha, q}=\left\|\Lambda(X)-\Lambda_{n}\left(X_{n}\right)\right\|_{\alpha, q} \leq\left\|\Lambda(X)-\Lambda_{n}(X)\right\|_{\alpha, q}+c\left\|X-X_{n}\right\|_{\alpha, q},
$$


and therefore

$$
\left\|X-X_{n}\right\|_{\alpha, q} \leq(1-c)^{-1}\left\|\Lambda(X)-\Lambda_{n}(X)\right\|_{\alpha, q} .
$$

Hence, in order to prove that $X_{n} \rightarrow X$ in $V_{\alpha}^{q}\left(\left[0, T_{0}\right] \times \Omega ; E\right)$, it suffices to prove that $\Lambda_{n}(X) \rightarrow \Lambda(X)$ in $V_{\alpha}^{q}\left(\left[0, T_{0}\right] \times \Omega ; E\right)$. But this follows from Lemma 4.4 and, picking $\lambda$ such that $\frac{1}{\tau}-\frac{1}{2}<\lambda<\alpha-\frac{1}{p}$, from Lemma 4.5 and the embedding of Lemma 3.8.

Step 2 We prove the result for general $T$.

Let $T_{0}$ as in Step 1. By Lemma 3.6, we have

$$
\left\|X-X_{n}\right\|_{V_{\alpha}^{q}\left(\left[0, \frac{3}{2} T_{0}\right] \times \Omega ; E\right)} \lesssim\left\|X-X_{n}\right\|_{V_{\alpha}^{q}\left(\left[0, T_{0}\right] \times \Omega ; E\right)}+\left\|X-X_{n}\right\|_{V_{\alpha}^{q}\left(\left[\frac{1}{2} T_{0}, \frac{3}{2} T_{0}\right] \times \Omega ; E\right)} .
$$

By Step 1, the first term on the right-hand side converges to 0 as $n \rightarrow \infty$. But so does the second term, noting that $X$ and $X_{n}$ are the unique solutions of the 'shifted' equations starting at initial time $\frac{1}{2} T_{0}$ with initial values $X\left(\frac{1}{2} T_{0}\right)$ and $X_{n}\left(\frac{1}{2} T_{0}\right)$ respectively.

Inductively, we obtain convergence in $V_{\alpha}^{q}\left(\left[0,\left(1+\frac{k}{2}\right) T_{0}\right] \times \Omega ; E\right)$ for all $k \in \mathbb{N}$ and hence in $V_{\alpha}^{q}([0, T] \times \Omega ; E)$ for all times $T$.

REMARK 4.6. Assume the hypotheses of Theorem 4.3 and additionally that $A_{n} \equiv$ $A$. Then

$$
X_{n} \rightarrow X \quad \text { in } V_{\alpha}^{p}([0, T] \times \Omega ; E)
$$

(rather than only in $V_{\alpha}^{q}([0, T] \times \Omega ; E)$ for $\left.1 \leq q<p\right)$. In particular, the solution of the equation with fixed coefficients $A, F$ and $G$ depends continuously on the initial datum $\xi \in L^{p}\left(\Omega, \mathscr{F}_{0} ; E\right)$ in the norm of $V_{\alpha}^{p}([0, T] \times \Omega ; E)$. This follows by repeating the proof of Theorem 4.3 and observing that this time parts (3) and (4) of Lemma 4.5 are not needed.

The second part of Theorem 4.3 asserts that $X_{n} \rightarrow X$ in $L^{q}(\Omega ; C([0, T] ; E))$. We will show next that the 'compensated solutions' even converge in the norm of $L^{q}\left(\Omega ; C^{\lambda}([0, T] ; E)\right)$.

THEOREM 4.7. Under the assumptions of Theorem 4.3, for all $0 \leq \lambda<\frac{1}{2}-\frac{1}{p}$, we have

$$
X_{n}-S_{n}(\cdot) \xi_{n} \rightarrow X-S(\cdot) \xi \quad \text { in } L^{q}\left(\Omega ; C^{\lambda}([0, T] ; E)\right)
$$

for all $1 \leq q<p$.

Proof. We may assume that $\frac{1}{\tau}-\frac{1}{2}<\lambda<\frac{1}{2}-\frac{1}{p}$. Choose $0<\alpha<\frac{1}{2}$ in such a way that $\frac{1}{\tau}-\frac{1}{2}<\lambda<\alpha-\frac{1}{p}$.

Let $\Lambda_{0}$ and $\Lambda_{n, 0}$ denote the Lipschitz continuous mappings used to solve (SCP) with data $(A, F, G, 0)$ and $\left(A_{n}, F_{n}, G_{n}, 0\right)$ respectively, i.e., they are given as in (4.4) with $\xi_{n} \equiv \xi=0$. We have

$$
\begin{aligned}
& \left\|X-S(\cdot) \xi-X_{n}+S_{n}(\cdot) \xi_{n}\right\|_{L^{q}\left(\Omega ; C^{\lambda}([0, T] ; E)\right)} \\
& \quad \leq\left\|\Lambda_{0}(X)-\Lambda_{n, 0}(X)\right\|_{L^{q}\left(\Omega ; C^{\lambda}([0, T] ; E)\right)}+\left\|\Lambda_{n, 0}(X)-\Lambda_{n, 0}\left(X_{n}\right)\right\|_{L^{q}\left(\Omega ; C^{\lambda}([0, T] ; E)\right)} .
\end{aligned}
$$


As a direct consequence of Lemma $4.5, \Lambda_{n, 0}(X) \rightarrow \Lambda_{0}(X)$ in $L^{q}\left(\Omega ; C^{\lambda}([0, T] ; E)\right)$.

Combining a standard factorization argument (e.g., as in the proof of [26, Theorem 6.2]) with the assumptions on $F_{n}$ and $G_{n}$, one sees that the mappings $\Lambda_{n, 0}$ are Lipschitz continuous from $V_{\alpha}^{p}([0, T] \times \Omega ; E)$ to $L^{q}\left(\Omega ; C^{\lambda}([0, T] ; E)\right)$, with uniformly bounded Lipschitz constants. Thus,

$$
\left\|\Lambda_{n, 0}(X)-\Lambda_{n, 0}\left(X_{n}\right)\right\|_{L^{q}\left(\Omega ; C^{\lambda}([0, T] ; E)\right)} \lesssim\left\|X-X_{n}\right\|_{\alpha, q} \rightarrow 0
$$

by Theorem 4.3. This finishes the proof.

\section{Applications}

\subsection{Approximating the noise}

As a first application, we show that if $H$ is separable, we may always approximate the cylindrical Brownian motion $W_{H}$ with finite dimensional noise. The strategy is to choose a sequence of projections $P_{n}$ on $H$ with finite dimensional ranges, which converges strongly to the identity. Then, we approximate the map $G$ by the functions $G_{n}:=G P_{n}$.

For $M$-type 2 spaces, such approximations were considered in [5]. In order to apply our results from the previous section, we must check that (G1) and (G2) hold.

Assumption (G1) follows from the ideal property of $\gamma$-radonifying operators and the uniform boundedness of the projections $P_{n}$. Assumption (G2) is an immediate consequence of [27, Proposition 2.4].

\subsection{Yosida approximations}

As we have already mentioned in the introduction, from a theoretical point of view, it is useful to be able to approximate the generator $A$ by its Yosida approximands $A_{n}:=n^{2} R(n, A)-n$.

For Hilbert spaces $E$, Yosida approximations for stochastic evolution equations are considered in Da Prato and Zabczyk [9] (see also [4] for an expanded argument), where continuous dependence in $L^{p}(\Omega ; C([0, T] ; E))$ is obtained without analyticity assumptions on $A$.

In order to apply our results, we must check that assumptions (A1) and (A2) hold for these operators. The uniform sectoriality (A1) follows from [13, Proposition 2.1.1 (f)]. As for the strong resolvent convergence (A2), we note that the standard proof of the Hille-Yosida theorem is to prove that the semigroups $\mathbf{S}_{n}$ generated by $A_{n}$ are uniformly exponentially bounded and converge strongly to the semigroup $\mathbf{S}$ generated by $A$. Taking Laplace transforms, the strong resolvent convergence follows. See also [2, Section 3.6]. 


\subsection{Approximating the coefficients in parabolic SPDEs}

In this section, we apply our results to stochastic partial differential equation. For simplicity, we confine ourselves to the situation where the nonlinearities $f$ and $g$ are time-independent and consider equations of the form

$$
\begin{cases}\frac{\partial u}{\partial t}(t, x)=\mathcal{A} u(t, x)+f(u(t, x))+\sum_{k=1}^{K} g_{k}(u(t, x)) \frac{\partial W_{k}}{\partial t}(t), & x \in \mathcal{O}, t>0, \\ u(t, x)=0, & x \in \partial \mathcal{O}, t>0, \\ u(0, x)=\xi(x), & x \in \mathcal{O} .\end{cases}
$$

Here, $\mathcal{O}$ is a bounded open domain in $\mathbb{R}^{d}$ and $\mathcal{A}$ is the second-order divergence form differential operator

$$
\mathcal{A} u(x)=\sum_{i=1}^{d} \frac{\partial}{\partial x_{i}}\left(a_{i j}(x) \sum_{j=1}^{d} \frac{\partial u}{\partial x_{j}}(x)\right)+\sum_{j=1}^{d} b_{j}(x) \frac{\partial u}{\partial x_{j}}(x) .
$$

The driving processes $W_{k}$ are independent real-valued standard Brownian motions. Under the assumptions of Theorem 1.1, we would like to approximate the coefficients $\mathbf{a}=\left(a_{i j}\right)$ and $\mathbf{b}=\left(b_{j}\right)$ as well as the functions $f$ and $g_{k}$ and study the convergence of the approximate solutions to the exact solution.

In order to reformulate the above SPDE as a stochastic Cauchy problem on the Banach space $L^{r}(\mathcal{O})$ (we use the exponent $r$ since the exponents $p$ and $q$ have already been used in a different meaning), we use a variational approach. Consider the sesquilinear form

$$
\mathfrak{a}[u, v]:=\int_{\mathcal{O}}(\mathbf{a} \nabla u) \cdot \overline{\nabla v}+(\mathbf{b} \cdot \nabla u) \bar{v} \mathrm{~d} x
$$

on the domain

$$
\mathrm{D}(\mathfrak{a}):=H_{0}^{1}(\mathcal{O}) .
$$

The sectorial operator $A$ on $L^{2}(\mathcal{O})$ associated with a generates a strongly continuous analytic semigroup $\mathbf{S}$, which by [10] extrapolates to a consistent family of strongly continuous analytic semigroups $\mathbf{S}^{(r)}$ on $L^{r}(\mathcal{O})$ for $1<r<\infty$. We denote their generators by $A^{(r)}$. Thus, $\mathbf{S}^{(2)}=\mathbf{S}$ and $A^{(2)}=A$. The forms $\mathfrak{a}_{n}$ and the associated semigroups $\mathbf{S}_{n}^{(r)}$ with generators $A_{n}^{(r)}$ are defined likewise.

LEMMA 5.1. If (i), (ii), and (iv) of Theorem 1.1 hold, then the operators $A^{(r)}$ and $A_{n}^{(r)}$ satisfy (A1) and (A2).

Proof. (A1): It follows from the uniform ellipticity and boundedness condition that the numerical ranges of the forms $\mathfrak{a}_{n}$ are contained in a common right open sector around the real axis. This in turn implies that there exists a constant $c \geq 0$ and an angle $\vartheta>0$ such that the shifted operators $A_{n}^{(2)}-c$ generate analytic semigroups $T_{n}^{(2)}(t)=e^{-c t} S_{n}^{(2)}(t)$ which are uniformly bounded on the sector $\Sigma_{\vartheta}:=\{z \in \mathbb{C} \backslash\{0\}:|\arg z|<\vartheta\}$. 
Now pick $1<s<\infty, s \neq 2$, such that $r$ lies between $s$ and 2 and put

$$
w:=2 \max \left\{s-1, s^{\prime}-1\right\} \kappa^{-1} C^{2} .
$$

Here, $s^{\prime}$ denotes the conjugate index to $s$, and $\kappa$ and $C$ are as in Theorem 1.1. It follows from [10, Theorem 5.1] that $\left\|S_{n}^{(s)}(t)\right\| \leq e^{w t}$ for all $t \geq 0$. By taking a larger value for $c$ if necessary, it follows that the semigroup $T_{n}^{(s)}(t)=$ $e^{-c t} S_{n}^{(s)}(t)$ satisfies $\left\|T_{n}^{(s)}(t)\right\| \leq 1$.

We are now in a position to use the Stein interpolation theorem in the version of [20, Lemma 5.8]. It follows that the semigroups $\mathbf{T}_{n}^{(r)}$ are uniformly bounded on a slightly smaller sector $\Sigma_{\vartheta^{\prime}}$. Rescaling again, this implies that the operators $A_{n}^{(r)}$ are uniformly sectorial.

(A2): It follows from (a special case of) [11, Theorem 5.2.4] that $A_{n}^{(2)} \rightarrow A^{(2)}$ in the strong resolvent sense. Since $\mathcal{O}$ is bounded, the embedding $H_{0}^{1}(\mathcal{O}) \hookrightarrow L^{2}(\mathcal{O})$ is compact. In particular, $A^{(2)}$ has compact resolvent. It now follows from [11, Theorem 4.3.4] that $A_{n}^{(r)} \rightarrow A^{(r)}$ in the strong resolvent sense.

To simplify notation, in what follows we fix $1<r<\infty$ and write $A:=A^{(r)}, A_{n}:=$ $A_{n}^{(r)}$, and $\mathbf{S}:=\mathbf{S}^{(r)}, \mathbf{S}_{n}:=\mathbf{S}_{n}^{(r)}$.

LEMMA 5.2. Assume that (iii) and (v) of Theorem 1.1 hold.

(1) The maps $F, F_{n}: L^{r}(\mathcal{O}) \rightarrow L^{r}(\mathcal{O})$ defined by

$$
[F(u)](x):=f(u(x)), \quad\left[F_{n}(u)\right](x):=f_{n}(u(x)),
$$

satisfy (F1) and (F2).

(2) The maps $G, G_{n}: L^{r}(\mathcal{O}) \rightarrow \mathscr{L}\left(\mathbb{R}^{K}, L^{r}(\mathcal{O})\right)$ defined by

$[G(u) h](x):=\sum_{k=1}^{K} g_{k}(u(x))\left[e_{k}, h\right], \quad\left[G_{n}(u) h\right](x):=\sum_{k=1}^{K} g_{n, k}(u(x))\left[e_{k}, h\right]$,

where $\left(e_{k}\right)_{k=1}^{K}$ is the standard unit basis of $\mathbb{R}^{K}$, satisfy $(\mathrm{G} 1)$ and $(\mathrm{G} 2)$.

Proof. (1) The assumptions imply that the maps $F$ and $F_{n}: L^{r}(\mathcal{O}) \rightarrow L^{r}(\mathcal{O})$ are Lipschitz continuous and of linear growth on $L^{r}(\mathcal{O})$ with uniform constants. Hence, (F1) is satisfied.

Assumption (F2) follows from dominated convergence.

(2) Let $T>0$ and $\mu$ be a finite Borel measure on $(0, T)$. If $\left(h_{m}\right)_{m \geq 1}$ is an orthonormal basis of $L^{2}((0, T), \mu)$, then $\left(h_{m} \otimes e_{k}\right)_{m \geq 1, k=1, \ldots, K}$ is an orthonormal basis of $L^{2}\left((0, T), \mu ; \mathbb{R}^{K}\right)$. Using this fact, it is easy to see that

$$
\|R\|_{\gamma\left(L^{2}\left((0, T), \mu ; \mathbb{R}^{K}\right), L^{r}(\mathcal{O})\right)} \leq \sum_{k=1}^{K}\left\|R e_{k}\right\|_{\gamma\left(L^{2}((0, T), \mu), L^{r}(\mathcal{O})\right)}
$$

for all $R \in \gamma\left(L^{2}\left((0, T), \mu ; \mathbb{R}^{K}\right), L^{r}(\mathcal{O})\right)$. 
This shows that it suffices to consider the case $K=1$. But in this case, we infer from [26, Example 5.5] that $G$ and $G_{n}$ are $\gamma$-Lipschitz continuous with a uniform $\gamma$-Lipschitz constant. Furthermore, by dominated convergence, $G_{n}(u) \rightarrow G(u)$ in $L^{r}(\mathcal{O})$ for all $u \in L^{r}(\mathcal{O})$.

We are now ready to rewrite our SPDE as an abstract Cauchy problem

$$
\begin{aligned}
d X(t) & =[A X(t)+F(X(t))] \mathrm{d} t+G(X(t)) \mathrm{d} W(t), \quad t \in[0, T], \\
X(0) & =\xi .
\end{aligned}
$$

We denote by $X_{n}$ the solution of this problem with $A, F, G, \xi$ replaced by $A_{n}, F_{n}, G_{n}, \xi_{n}$. Noting that the type of $L^{r}(\mathcal{O})$ is $\min \{r, 2\}$, we deduce the following strengthened version of Theorem 1.1 from Theorem 4.7:

THEOREM 5.3. Assume that (i) - (v) of Theorem 1.1 hold, and let $1<r<$ $\infty$ and $p>\max \left\{2, r^{\prime}\right\}$, where $r^{\prime}$ denotes the conjugate index to $r$. If $\xi_{n} \rightarrow \xi$ in $L^{p}\left(\Omega, \mathscr{F}_{0} ; L^{r}(\mathcal{O})\right)$, then

$$
X_{n}-S_{n}(\cdot) \xi_{n} \rightarrow X-S(\cdot) \xi \text { in } L^{q}\left(\Omega ; C^{\lambda}\left([0, T] ; L^{r}(\mathcal{O})\right)\right.
$$

for all $1 \leq q<p$ and $0 \leq \lambda<\frac{1}{2}-\frac{1}{p}$. Moreover, $X_{n} \rightarrow X$ in $L^{q}\left(\Omega ; C\left([0, T] ; L^{r}(\mathcal{O})\right)\right)$ for all $1 \leq q<p$.

We emphasize that the applicability of our approach is by no means limited to second-order differential operators in divergence form and Dirichlet boundary conditions. Indeed, our approach applies to any sequence of operators for which the conditions (A1) and (A2) can be verified. This includes, for instance, the case of Neumann boundary conditions in the above example (provided $\partial \mathcal{O}$ is Lipschitz, so as to ensure the compactness of the embedding $H^{1}(\mathcal{O}) \hookrightarrow L^{2}(\mathcal{O})$ ).

\subsection{Approximating the domain}

A map $\mathbf{S}:[0, \infty) \rightarrow \mathscr{L}(E)$ is called a degenerate semigroup if it is strongly continuous and we have $S(t+s)=S(t) S(s)$ for all $t, s \geq 0$. Thus, the only difference to a strongly continuous semigroup is that we do not assume that $S(0)=I$. Instead, $\pi:=S(0)$ is now a bounded projection that commutes with every operator $S(t)$. Consequently, we can write $S(t)=\imath \tilde{S}(t) \pi$, where $\pi$ is the projection viewed as an operator onto its range $\tilde{E}, \tilde{\mathbf{S}}$ is the restriction of $\mathbf{S}$ to $\tilde{E}$ which is invariant under $\mathbf{S}$, and $\iota: \tilde{E} \subseteq E$ is the canonical inclusion map.

It is easy to see that the Laplace transform of $\mathbf{S}$ is given by $\iota R(\lambda, \tilde{A}) \pi$ for $\operatorname{Re} \lambda$ large enough, where $R(\lambda, \tilde{A})$ denotes the resolvent of the generator $\tilde{A}$ of the restricted semigroup $\tilde{\mathbf{S}}$. We may thus say that the generator $A$ of $\mathbf{S}$ is the operator $\tilde{A}$, viewed as an operator on $E$. As a replacement for the resolvent of $A$, we define $R_{\lambda}(A):=\imath R(\lambda, \tilde{A}) \pi$ and note that $R_{\lambda}(A)$ is a pseudo-resolvent. We will call a degenerate semigroup $\mathbf{S}$ analytic if the restricted semigroup $\tilde{\mathbf{S}}$ is analytic on $\tilde{E}$. For more information on degenerate semigroups and pseudo-resolvents, we refer the reader to [1]. 
When we allow degenerate semigroups, we will assume that $A_{n}$ and $A$ are generators of analytic, degenerate semigroups $\mathbf{S}_{n}:=\iota_{n} \tilde{\mathbf{S}}_{n} \pi_{n}$ and $\mathbf{S}:=\imath \tilde{\mathbf{S}} \pi$. Furthermore, we will make the following assumptions:

(A1') The operators $A$ and $A_{n}$ are uniformly sectorial in the sense there exist numbers $M \geq 1$ and $w \in \mathbb{R}$ such that

(i) $\{\lambda \in \mathbb{C}: \operatorname{Re} \lambda>w\}$ is contained in $\rho(\tilde{A}) \cap\left(\bigcap_{n} \rho\left(\tilde{A}_{n}\right)\right)$, where $\tilde{A}$ and $\tilde{A}_{n}$ denote the generators of the strongly continuous semigroups $\tilde{\mathbf{S}}$ and $\tilde{\mathbf{S}}_{n}$ respectively.

(ii) We have

$$
\sup _{\operatorname{Re} \lambda>w}\left\|(\lambda-w) R_{\lambda}\left(A_{n}\right)\right\| \leq M, \sup _{\operatorname{Re} \lambda>w}\left\|(\lambda-w) R_{\lambda}(A)\right\| \leq M .
$$

(A2') The operators $A_{n}$ converge to $A$ in the strong resolvent sense, i.e.

$$
\lim _{n \rightarrow \infty} R_{\lambda}\left(A_{n}\right) x=R_{\lambda}(A) x
$$

for all $\operatorname{Re} \lambda>w$ and $x \in E$.

The reader may check that, mutatis mutandis, all results of this article extend to the degenerate case when replacing (A1) and (A2) with (A1') and (A2'). In particular, there is a Trotter-Kato type theorem for degenerate semigroups, see [1, Theorem 5.1].

As an application, we shall use degenerate semigroups to study the dependence of the solutions of the SPDE in the previous section on the domain $\mathcal{O}$. Recall that a sequence of domains $\mathcal{O}_{n}$ is said to converge toward $\mathcal{O}$ in the sense of Mosco

- If $\left(u_{n}\right)$ is a sequence in $H_{0}^{1}\left(\mathbb{R}^{d}\right)$ with $u_{n} \in H_{0}^{1}\left(\mathcal{O}_{n}\right)$ for all $n$, then every weak limit point of this sequence in $H_{0}^{1}\left(\mathbb{R}^{d}\right)$ lies in $H_{0}^{1}(\mathcal{O})$;

- For all $u \in H_{0}^{1}(\mathcal{O})$, there exists a sequence $\left(u_{n}\right)$ is a sequence in $H_{0}^{1}\left(\mathbb{R}^{d}\right)$ with $u_{n} \in H_{0}^{1}\left(\mathcal{O}_{n}\right)$ for all $n$ such that $u_{n} \rightarrow u$ in $H^{1}\left(\mathbb{R}^{d}\right)$.

We give two examples of Mosco convergence. We refer to [11] for the proofs and further examples.

(1) If $\mathcal{O}_{1} \subseteq \mathcal{O}_{2} \subseteq \cdots$ and $\bigcup_{n \geq 1} \mathcal{O}_{n}=\mathcal{O}$, then $\mathcal{O}_{n} \rightarrow \mathcal{O}$ in the sense of Mosco.

(2) If $\mathcal{O}_{1} \supseteq \mathcal{O}_{2} \supseteq \cdots$ and $\bigcap_{n \geq 1} \mathcal{O}_{n}=\mathcal{O}$, then $\mathcal{O}_{n} \rightarrow \mathcal{O}$ in the sense of Mosco provided $\mathcal{O}$ satisfies the regularity condition

$$
H_{0}^{1}(\mathcal{O})=\left\{u \in H^{1}\left(\mathbb{R}^{d}\right): u \equiv 0 \text { a.e. on } \complement \overline{\mathcal{O}}\right\}
$$

For simplicity, we assume that a single set of coefficients $\mathbf{a}$ and $\mathbf{b}$ is given, defined on all of $\mathbb{R}^{d}$ and satisfying assumptions (i) and (ii) of Theorem 1.1. Given an open set $\mathcal{O} \subseteq \mathbb{R}^{d}$, we may again consider the form $\mathfrak{a}$ defined by (5.1). Associated with this form, we obtain, as before, a strongly continuous analytic semigroup $\mathbf{S}$ on $L^{r}(\mathcal{O})$. We may consider $\mathbf{S}$ as a degenerate semigroup on $L^{r}\left(\mathbb{R}^{d}\right)$ by extending functions in $L^{r}(\mathcal{O})$ identically 0 outside $\mathcal{O}$. Its (not necessarily densely defined) generator is denoted by $A$. 
Suppose next that we are given a sequence of domains $\mathcal{O}_{n}$ converging to $\mathcal{O}$ in the sense of Mosco and consider the corresponding forms $\mathfrak{a}_{n}$ on $H_{0}^{1}\left(\mathcal{O}_{n}\right)$. The associated degenerate semigroups on $L^{r}\left(\mathbb{R}^{d}\right)$ are denoted by $\mathbf{S}_{n}$ and their (not necessarily densely defined) generators by $A_{n}$. It is clear from the results of Sect. 5.3 that condition ( $\left.\mathrm{A}^{\prime}\right)$ is satisfied. We thus have to verify the (pseudo-)resolvent convergence (A2 ${ }^{\prime}$ ). From Theorems 5.2.6 and 4.3.4 of [11], we infer:

LEMMA 5.4. Let $\mathcal{O}$ and $\mathcal{O}_{n}$ be bounded open domains contained in some fixed bounded subset of $\mathbb{R}^{d}$ and suppose that $\mathcal{O}_{n} \rightarrow \mathcal{O}$ in the sense of Mosco. If (i) and (ii) of Theorem 1.1 hold, then for all $1<r<\infty$ we have $A_{n} \rightarrow A$ in the strong resolvent sense.

As before, we further assume that the functions $f, g: \mathbb{R} \rightarrow \mathbb{R}$ satisfy the assumptions (iii) and (v) of Theorem 1.1 and that the driving processes $W_{k}$ are independent real-valued standard Brownian motions. We have the following result.

THEOREM 5.5. Let $1<r<\infty$ and $p>\max \left\{2, r^{\prime}\right\}$ and suppose that the initial datum $\xi$ belongs to $L^{p}\left(\Omega, \mathscr{F}_{0} ; L^{r}\left(\mathbb{R}^{d}\right)\right)$. Under the above assumptions, let

$$
X:=\operatorname{sol}(A, F, G, \xi), \quad X_{n}:=\operatorname{sol}\left(A_{n}, F, G, \xi\right),
$$

denote the unique mild solutions of the associated stochastic evolution equations. Then,

$$
X_{n}-S_{n}(\cdot) \xi \rightarrow X-S(\cdot) \xi \text { in } L^{q}\left(\Omega ; C^{\lambda}\left([0, T] ; L^{r}\left(\mathbb{R}^{d}\right)\right)\right)
$$

for all $1 \leq q<p$ and $0 \leq \lambda<\frac{1}{2}-\frac{1}{p}$. Moreover, $X_{n} \rightarrow X$ in $L^{q}\left(\Omega ; C\left([0, T] ; L^{r}(\mathcal{O})\right)\right)$ for all $1 \leq q<p$.

Once again, it is possible to approximate simultaneously the domain $\mathcal{O}$, the coefficients $\mathbf{a}, \mathbf{b}$, the nonlinearities $f$ and $g$, and the initial datum $\xi$. We leave the details to the interested reader.

\section{Acknowledgments}

The authors are grateful to Marta Sanz-Solé for pointing out the references [3,24] and Mark Veraar for a helpful discussion.

Open Access. This article is distributed under the terms of the Creative Commons Attribution Noncommercial License which permits any noncommercial use, distribution, and reproduction in any medium, provided the original author(s) and source are credited.

\section{REFERENCES}

[1] W. ARENDT, Approximation of degenerate semigroups. Taiwanese J. Math. 5 (2001), 279-295.

[2] W. ARENDT, C. J. K. BAtTy, M. Hieber, AND F. NeUBRANDER, Vector-valued Laplace transforms and Cauchy problems, vol. 96 of Monographs in Mathematics, Birkhäuser Verlag, Basel, 2001. 
[3] V. Bally, A. Millet, AND M. SAnZ- SolÉ, Approximation and support theorem in Hölder norm for parabolic stochastic partial differential equations. Ann. Probab. 23 (1995), 178-222.

[4] J. BIERKENS, Long term dynamics of stochastic evolution equations, PhD thesis, University of Leiden, 2010.

[5] Z. BRZEŹNIAK, On stochastic convolution in Banach spaces and applications. Stochastics Stochastics Rep. 61 (1997), 245-295.

[6] Z. BRZEŹNIAK AND K. D. ELWORTHY, Stochastic differential equations on Banach manifolds. Methods Funct. Anal. Topology, 6 (2000), 43-84.

[7] P. Clément, B. De Pagter, F. A. Sukochev, And H. Witvliet, Schauder decompositions and multiplier theorems. Studia Math., 138 (2000), 135-163.

[8] G. DA PRATO, S. KWAPIEN, AND J. ZABCZYK, Regularity of solutions of linear stochastic equations in Hilbert spaces. Stochastics, 23 (1987), 1-23.

[9] G. DA PRATO AND J. ZABCZYK, Stochastic equations in infinite dimensions, vol. 44 of Encyclopedia of Mathematics and its Applications, Cambridge University Press, Cambridge, 1992.

[10] D. DANERS, Heat kernel estimates for operators with boundary conditions. Math. Nachr., 217 (2000), 13-41.

[11] D. DANERS, Domain perturbation for linear and semi-linear boundary value problems, in Handbook of Differential Equations, vol. 6, North-Holland, 2008, pp. 1-81.

[12] J. Dettweiler, L. W. Weis, And J. M. A. M. V. NeERVEN, Space-time regularity of solutions of the parabolic stochastic Cauchy problem. Stoch. Anal. Appl., 24 (2006), 843-869.

[13] M. H. A. HAASE, The functional calculus for sectorial operators, vol. 169 of Operator Theory: Advances and Applications, Birkhäuser Verlag, Basel, 2006.

[14] J. HoFFMANN- J $\varnothing$ RGENSEN, Sums of independent Banach space valued random variables. Studia Math., 52 (1974), 159-186.

[15] W. B. JOHNSON AND J. LindENSTRAUSS, eds., Handbook of the geometry of Banach spaces. Vol. I, North-Holland Publishing Co., Amsterdam, 2001.

[16] W. B. JOHNSON AND J. LinDENSTRAUSS, eds., Handbook of the geometry of Banach spaces. Vol. 2, North-Holland Publishing Co., Amsterdam, 2003.

[17] O. Kallenberg, Foundations of modern probability, Probability and its Applications, SpringerVerlag, New York, second ed., 2002.

[18] N. J. KALtON AND L. W. WeIs, The $H^{\infty}$-calculus and square function estimates. Preprint, 2004.

[19] N. KRYLOv, An analytic approach to SPDEs, in in: "Stochastic Partial Differential Equations: Six Perspectives, vol. 64 of Math. Surveys Monogr., Amer. Math. Soc., Providence, RI, 1999, pp. 185-242.

[20] P. C. KunstManN AND L. W. WeIs, Maximal $L_{p}$-regularity for parabolic equations, Fourier multiplier theorems and $H^{\infty}$-functional calculus, in Functional analytic methods for evolution equations, vol. 1855 of Lecture Notes in Math., Springer, Berlin, 2004, pp. 65-311.

[21] M. C. KunZE AND J. M. A. M. V. NeERVEN. Work in progress, 2011.

[22] S. KWAPIEN, On Banach spaces containing $c_{0}$. Studia Math. 52 (1974), 187-188. A supplement to the paper by J. Hoffmann-Jørgensen: "Sums of independent Banach space valued random variables" (Studia Math. 52 (1974), 159-186).

[23] C. MARINElLI, C. PRÉVÔT, AND M. RÖCKNER, Regular dependence on initial data for stochastic evolution equations with multiplicative Poisson noise. J. Funct. Anal. 258 (2010), 616-649.

[24] A. MiLlET AND M. SANZ- SOLÉ, A simple proof of the support theorem for diffusion processes, in Séminaire de Probabilités, XXVIII, vol. 1583 of Lecture Notes in Math., Springer, Berlin, 1994, pp. 36-48.

[25] J.M.A.M.v. NEERVEN, $\gamma$-Radonifying operators: a survey. Proceedings of the CMA, 44 (2010), $1-62$.

[26] J.M.A.M.V. NEERVEN, M. C. VERAAR, AND L. WEIS, Stochastic evolution equations in UMD Banach spaces. J. Funct. Anal., 255 (2008), 940-993.

[27] J.M.A.M.v. NEERVEN, M. C. VeraAR, AND L. W. WEIS, Stochastic integration in UMD Banach spaces. Ann. Probab., 35 (2007), 1438-1478.

[28] J.M.A.M.V. NEERVEN AND L. W. WEIS, Stochastic integration of functions with values in a Banach space. Studia Math., 166 (2005), 131-170. 
[29] S. PESZAT AND J. ZABCZYK, Strong Feller property and irreducibility for diffusions on Hilbert spaces. Ann. Probab., 23 (1995), 157-172.

[30] J. SEIDLER, Weak convergence of infinite-dimensional diffusions. Stochastic Anal. Appl., 15 (1997), $399-417$.

[31] L. W. WEIS, Operator-valued Fourier multiplier theorems and maximal $L_{p}$-regularity. Math. Ann., 319 (2001), 735-758.

M. Kunze AND J. van Neerven

Delft Institute of Applied Mathematics

Delft University of Technology

P.O. Box 5031, 2600 GA Delft, The Netherlands

E-mail:M.C.Kunze@TUDelft.nl,J.M.A.M.vanNeerven@TUDelft.nl 